\title{
Novel Robust Exponential Stability of Markovian Jumping Impulsive Delayed Neural Networks of Neutral-Type with Stochastic Perturbation
}

\author{
Yang Fang, ${ }^{1}$ Kelin Li, ${ }^{1,2}$ and Yunqi Yan ${ }^{3}$ \\ ${ }^{1}$ School of Science, Sichuan University of Science \& Engineering, Sichuan 643000, China \\ ${ }^{2}$ Institute of Nonlinear Physical Science, Sichuan University of Science \& Engineering, Sichuan 643000, China \\ ${ }^{3}$ College of Mechanical Engineering, Sichuan University of Science \& Engineering, Sichuan 643000, China
}

Correspondence should be addressed to Yang Fang; fyang22@163.com

Received 28 January 2016; Revised 12 April 2016; Accepted 19 May 2016

Academic Editor: Olfa Boubaker

Copyright (C) 2016 Yang Fang et al. This is an open access article distributed under the Creative Commons Attribution License, which permits unrestricted use, distribution, and reproduction in any medium, provided the original work is properly cited.

The robust exponential stability problem for a class of uncertain impulsive stochastic neural networks of neutral-type with Markovian parameters and mixed time-varying delays is investigated. By constructing a proper exponential-type LyapunovKrasovskii functional and employing Jensen integral inequality, free-weight matrix method, some novel delay-dependent stability criteria that ensure the robust exponential stability in mean square of the trivial solution of the considered networks are established in the form of linear matrix inequalities (LMIs). The proposed results do not require the derivatives of discrete and distributed time-varying delays to be 0 or smaller than 1 . Moreover, the main contribution of the proposed approach compared with related methods lies in the use of three types of impulses. Finally, two numerical examples are worked out to verify the effectiveness and less conservativeness of our theoretical results over existing literature.

\section{Introduction}

Up to now, the stability analysis of neural networks is an important research field in modern cybernetic area, since most of the successful applications of neural networks significantly depend on the stability of the equilibrium point of neural networks. Many papers related to this problem have been published in the literature; see [1] for a survey.

During implementation of artificial neural networks, time-varying delays [2-4] are unavoidable due to finite switching speeds of the amplifiers, and the neural signal propagation is often distributed in a certain time period with the presence of an amount of parallel pathways with a variety of axon sizes and lengths. Therefore, it is necessary to consider mixed time-varying delays (discrete time-varying delay and distributed time-varying delay) to design the neural networks models. There are many works focusing on the mixed timevarying delays [5-8], among which delay-dependent criteria are generally less conservative than delay-independent ones when the sizes of time-delays are small, and the maximum allowable delay bound is the main performance index of delay-dependent stability analysis [9]. In addition, as a special type of time delayed neural networks, neutral-type neural networks precisely describe that the past state of the networks will affect the current state. Therefore, the problems of stability and synchronization for such a class of neural networks have been studied in many references; see [10-22].

It is well known that the other three sources which may lead to instability and poor performances in neural networks are stochastic perturbation, impulsive perturbations, and parametric uncertainties. Most of this viewpoint is attributable to the following three reasons: (1) A neural network can be stabilized or destabilized by certain stochastic inputs [23-26]. (2) In the real world, many evolutionary processes are characterized by abrupt changes at time. These changes are called impulsive phenomena, which have been found in various fields, such as physics, optimal control, and biological mathematics [27]. (3) The effects of parametric uncertainties cannot be ignored in many applications [2830]. Hence, stochastic perturbation, impulsive perturbations, 
and parametric uncertainties also should be taken into consideration when dealing with the stability issue of neural networks.

On the other hand, Markovian jumping systems [31] can be seen as a special class of hybrid systems with two different states, which involve both time-evolving and event-driven mechanisms. So such systems would be used to model the abrupt phenomena such as random failures and repairs of the components, changes in the interconnections of subsystems, and sudden environment changes. Thus, many relevant analysis results for Markovian jumping neural networks with impulses have been reported; see [32-38] and the references therein.

Recently, by using the concept of the minimum impulsive interval, Bao and Cao [11], Zhang et al. [12], and Gao et al. [13] derived some sufficient conditions to ensure exponential stability in mean square for neutral-type impulsive stochastic neural networks with Markovian jumping parameters and mixed time delays. However, in [11-13], the authors ignored parametric uncertainties. And in these three papers, the derivatives of time-varying delays need to be zero or smaller than one. So far, there are few results on the study of robust exponential stability of neutral-type impulsive stochastic neural networks with Markovian jumping parameters, mixed time-varying delays, and parametric uncertainties. More importantly, the impulses can be divided into three types to discuss the following: the impulses are stabilizing; the impulses are neutral-type (i.e., they are neither helpful for stability of neural networks nor destabilizing); and the impulses are destabilizing. Some interesting results for analyzing and synthesizing impulsive nonlinear systems that divide impulses into three types can be seen in [39-46]. In [39$41,43]$, the authors studied the stability problem of impulsive neural networks with discrete time-varying delay by using the Lyapunov-Razumikhin method; several criteria for global exponential stability of the discrete-time or continuous-time neural networks are established in terms of matrix inequalities. In [42, 44-46], combining the impulsive comparison theory and triangle inequality, some important results about three-type impulses for different neural networks have been obtained. However, distributed time-varying delay has not been taken into account in all abovementioned references; how to deal with the stability problem of Markovian jumping impulsive stochastic neural networks with mixed delays is also a meaningful direction. Motivated by above discussion, based on the concepts of three-type impulses, this paper focuses on the robust exponential stability in mean square of impulsive stochastic neural networks with Markovian jumping parameters, mixed time-varying delays, and parametric uncertainties. By constructing a proper exponentialtype Lyapunov-Krasovskii functional, linear matrix inequality (LMI) technique, Jensen integral inequality and freeweight matrix method, several novel sufficient conditions in terms of linear matrix inequalities (LMIs) are derived to guarantee the robust exponential stability in mean square of the trivial solution of the considered model. Compared with references [11-13], the constructed model renders more practical factors since the parametric uncertainties have been taken into account, and the derivatives of discrete and distributed time-varying delays need to be 0 or smaller than 1. Moreover, the main contribution of the proposed approach compared with related methods lies in the use of three types of impulses.

The organization of this paper is as follows. In Section 2, the robust exponential stability problem of impulsive stochastic neural networks with Markovian jumping parameters, mixed time-varying delays, and parametric uncertainties is described and some necessary definitions and lemmas are given. Some new robust exponential stability criteria are obtained in Section 3. In Section 4, two illustrative examples are given to show the effectiveness and less conservatism of the proposed method. Finally, conclusions are given in Section 5.

Notation. Let $\mathbb{R}$ denote the set of real numbers, let $\mathbb{R}^{+}$denote the set of all nonnegative real numbers, let $\mathbb{R}^{n}$ and $\mathbb{R}^{n \times m}$ denote the $n$-dimensional and $n \times m$ dimensional real spaces equipped with the Euclidean norm, and let $\|\cdot\|$ refer to the Euclidean vector norm and the induced matrix norm. $\mathbb{N}^{+}$denotes the set of positive integers. For any matrix $X \in$ $\mathbb{R}^{n \times n}, X>0$ denotes that $X$ is a symmetric and positive definite matrix. If $X_{1}, X_{2}$ are symmetric matrices, then $X_{1} \leq X_{2}$ means that $X_{1}-X_{2}$ is a negative semidefinite matrix. $X^{T}$ and $X^{-1}$ mean the transpose of $X$ and the inverse of a square matrix. $I$ denotes the identity matrix with appropriate dimensions. Let $\tau>0$ and $C\left([-\tau, 0] ; \mathbb{R}^{n}\right)$ denote the family of all continuous $\mathbb{R}^{n}$-valued functions $\xi(\theta)$ on $[-\tau, 0]$ with the norm $|\xi|=\sup _{-\tau \leq \theta \leq 0}\|\xi(\theta)\|$. Let $\omega(t)=\left[\omega_{1}(t), \omega_{2}(t), \ldots, \omega_{n}(t)\right]^{T}$ be an $n$-dimensional Brownian motion defined on a complete probability space $(\Omega, \mathscr{F}, \mathscr{P})$ with a natural filtration $\left\{\mathscr{F}_{t}\right\}_{t \geq 0}$ (i.e., $\mathscr{F}_{t}=\sigma\{\omega(s)$ : $0 \leq s \leq t\}$ ), which satisfies $E d \omega(t)=0$ and $E[d \omega(t)]^{2}=$ dt. $L_{\mathscr{F}_{t}}^{p}\left([-\tau, 0] ; \mathbb{R}^{n}\right)(t \geq 0)$ denote the family of all $\mathscr{F}_{t}$ measurable bounded $C\left([-\tau, 0] ; \mathbb{R}^{n}\right)$-valued random variables $\xi=\{\xi(\theta):-\tau \leq \theta \leq 0\}$ such that $\int_{-\tau}^{0} E|\xi(s)|^{p} d s<\infty$, where $E\{\cdot\}$ stands for the correspondent expectation operator with respect to the given probability measure $\mathscr{P}$. The notation $\star$ always denotes the symmetric block in one symmetric matrix. Matrix dimensions, if not explicitly stated, are assumed to be compatible for operations.

\section{Model Description and Preliminaries}

Let $\{r(t), t \geq 0\}$ be a right continuous Markov chain in a complete probability space $(\Omega, \mathscr{F}, \mathscr{P})$ taking values in a finite state space $S=\{1,2, \ldots, N\}$ with generator $\Pi=\left(\pi_{i j}\right)_{N \times N}$ given by

$$
\begin{aligned}
& \mathscr{P}\{r(t+\Delta t)=j \mid r(t)=i\} \\
&= \begin{cases}\pi_{i j} \Delta t+o(\Delta t), & \text { if } i \neq j, \\
1+\pi_{i i} \Delta t+o(\Delta t), & \text { if } i=j,\end{cases}
\end{aligned}
$$

where $\Delta t>0$ and $\lim _{\Delta t \rightarrow 0}\left(o(\Delta t / \Delta t)=0\right.$. Here $\pi_{i j} \geq 0(i \neq$ $j)$ is the transition rate from mode $i$ to mode $j$ while $\pi_{i i}=$ $-\sum_{j \neq i} \pi_{i j}$ is the transition rate from mode $i$ to mode $i$. 
Consider a class of impulsive stochastic neural networks of neural-type with Markovian jumping parameters, mixed time-varying delays, and parametric uncertainties, which can be presented by the following impulsive integrodifferential equation:

$$
\begin{aligned}
& d\left[u(t)-D(r(t)) u\left(t-\tau_{3}(t)\right)\right]=[-C(r(t)) u(t) \\
& \quad+(A(r(t))+\Delta A(r(t))) f(u(t)) \\
& \quad+(B(r(t))+\Delta B(r(t))) f\left(u\left(t-\tau_{1}(t)\right)\right) \\
& \left.\quad+(E(r(t))+\Delta E(r(t))) \int_{t-\tau_{2}(t)}^{t} f(u(s)) d s+J\right] d t \\
& \quad+\sigma\left(t, r(t), u(t), u\left(t-\tau_{1}(t)\right), u\left(t-\tau_{2}(t)\right),\right. \\
& \left.\quad u\left(t-\tau_{3}(t)\right)\right) d \omega(t), \quad t \neq t_{k}, \\
& \mathscr{D u}\left(t_{k}^{+}\right)=W_{k}(r(t)) \mathscr{D} u\left(t_{k}^{-}\right), \quad t=t_{k}, k \in N^{+},
\end{aligned}
$$

for $t>0$, where $u(t)=\left(u_{1}(t), u_{2}(t), \ldots, u_{n}(t)\right)^{T} \in \mathbb{R}^{n}$ is the state vector associated with $n$ neurons at time $t$. In the continuous part of system (2), $C(r(t))=\operatorname{diag}\left\{c_{1}(r(t))\right.$, $\left.c_{2}(r(t)), \ldots, c_{n}(r(t))\right\}$ is a diagonal matrix with positive entries $c_{i}(r(t))>0(i=1,2, \ldots, n)$; the matrices $A(r(t))=$ $\left(a_{i j}(r(t))\right)_{n \times n}, B(r(t))=\left(b_{i j}(r(t))\right)_{n \times n}$, and $E(r(t))=$ $\left(e_{i j}(r(t))\right)_{n \times n}$ are the connection weight matrix, the discrete time-varying delay connection weight matrix, and the distributed-delay connection weight matrix, respectively; $\Delta A(r(t)), \Delta B(r(t))$, and $\Delta D(r(t))$ are the time-varying parametric uncertainties; $f(u(t))=\left(f_{1}\left(u_{1}(t)\right), f_{2}\left(u_{2}(t)\right), \ldots\right.$, $\left.f_{n}\left(u_{n}(t)\right)\right)^{T} \in \mathbb{R}^{n}$ is the nonlinear neuron activation function which describes the behavior in which the neurons respond to each other; $J=\left[J_{1}, J_{2}, \ldots, J_{n}\right]^{T} \in \mathbb{R}^{n}$ is a constant external input vector; $\tau_{1}(t), \tau_{2}(t)$, and $\tau_{3}(t)$ are, namely, the discrete time-varying delay, distributed time-varying delay, and neutral time-varying delay, which satisfy $0 \leq h_{1} \leq \tau_{1}(t) \leq$ $h_{2}, \dot{\tau}_{1}(t) \leq \mu_{1}, 0 \leq \tau_{2}(t) \leq \tau_{2}, \dot{\tau}_{2}(t) \leq \mu_{2}, 0 \leq \tau_{3}(t) \leq \tau_{3}$, and $\dot{\tau}_{3}(t) \leq \mu_{3}$; the noise perturbation (or the diffusion coefficient) $\sigma\left(t, r(t), u(t), u\left(t-\tau_{1}(t)\right), u\left(t-\tau_{2}(t)\right), u\left(t-\tau_{3}(t)\right)\right)$ : $\mathbb{R}^{+} \times S \times \mathbb{R}^{n} \times \mathbb{R}^{n} \times \mathbb{R}^{n} \rightarrow \mathbb{R}^{n \times n}$ is a Borel measurable function. In the discrete part of system (2), $\mathscr{D} u\left(t_{k}^{+}\right)=W_{k}(r(t)) \mathscr{D} u\left(t_{k}^{-}\right)$, $k \in \mathbb{N}^{+}$is the impulse at the moment of time $t_{k}$ of an operator defined as $\mathscr{D} u(t)=u(t)-D(r(t)) u\left(t-\tau_{3}(t)\right) ; W_{k}(r(t)) \in \mathbb{R}^{n \times n}$ is the impulse gain matrix at the moment of time $t_{k}$; the discrete instant set $\left\{t_{k}\right\}$ satisfies $0=t_{0}<t_{1}<t_{2}<\cdots<t_{k}<$ $\cdots, \lim _{k \rightarrow \infty} t_{k}=\infty ; \mathscr{D} u\left(t_{k}^{-}\right)$and $\mathscr{D} u\left(t_{k}^{+}\right)$are the left-hand and right-hand limits of operator $\mathscr{D} u(t)$ at $t_{k}$, respectively; as usual, we always assume that $\mathscr{D} u\left(t_{k}^{+}\right)=\mathscr{D} u\left(t_{k}\right)$.

Remark 1. In the continuous part of system (2), the evolution of state vector $u(t)$ is driven by the evolution of the operator $\mathscr{D} u(t)=u(t)-D(r(t)) u\left(t-\tau_{3}(t)\right)$. Consequently, we consider state jumping of the operator $\mathscr{D} u(t)$ at impulsive time in the discrete part of system (2). In system (2) of [13], $\dot{\omega}(t)$ has been used to build the main model, which is wrong since Brown motion is nowhere differentiable with probability 1 [47].
For convenience, we denote $r(t)=i, i \in S$; then the matrices $D(r(t)), C(r(t)), A(r(t)), B(r(t)), E(r(t)), \Delta A(r(t))$, $\Delta B(r(t))$, and $\Delta E(r(t))$ will be written as $D_{i}, C_{i}, A_{i}, B_{i}, E_{i}$, $\Delta A_{i}, \Delta B_{i}$, and $\Delta E_{i}$, respectively. Therefore, system (2) can be rewritten as follows:

$$
\begin{gathered}
d\left[u(t)-D_{i} u\left(t-\tau_{3}(t)\right)\right]=\left[-C_{i} u(t)+\left(A_{i}+\Delta A_{i}\right)\right. \\
\cdot f(u(t))+\left(B_{i}+\Delta B_{i}\right) f\left(u\left(t-\tau_{1}(t)\right)\right) \\
\left.+\left(E_{i}+\Delta E_{i}\right) \int_{t-\tau_{2}(t)}^{t} f(u(s)) d s+J\right] d t+\sigma(t, i, \\
\left.u(t), u\left(t-\tau_{1}(t)\right), u\left(t-\tau_{2}(t)\right), u\left(t-\tau_{3}(t)\right)\right) d \omega(t), \\
\mathscr{D} u\left(t_{k}^{+}\right)=W_{i k} \mathscr{D} u\left(t_{k}^{-}\right), \quad t=t_{k}, k \in N^{+},
\end{gathered}
$$

The initial condition of system (3) is given in the following form:

$$
\begin{aligned}
u(s) & =\varphi(s), \quad s \in[-\tau, 0], \\
r(0) & =i_{0}, \\
\tau & =\max \left\{h_{2}+\tau_{3}, \tau_{2}+\tau_{3}\right\},
\end{aligned}
$$

for any $\varphi(s) \in L_{\mathscr{F}_{0}}^{2}\left([-\tau, 0] ; \mathbb{R}^{n}\right)$.

To prove our main results, the following hypotheses are needed:

(H1) All the eigenvalues of matrix $D_{i}, i \in S$, are inside the unit circle, which guarantees the stability of difference system $u(t)-D_{i} u\left(t-\tau_{3}(t)\right)=0$.

$(H 2)$ Each neuron activation function $f_{j}$ is continuous [48], and there exist scalars $l_{j}^{-}$and $l_{j}^{+}$such that

$$
l_{j}^{-} \leq \frac{f_{j}(a)-f_{j}(b)}{a-b} \leq l_{j}^{+},
$$

for any $a, b \in \mathbb{R}, a \neq b, j=1,2, \ldots, n$, where $l_{j}^{+}$and $l_{j}^{-}$ can be positive, negative, or zero. And we set

$$
\begin{aligned}
& L_{1}=\operatorname{diag}\left(l_{1}^{-}, l_{2}^{-}, \ldots, l_{n}^{-}\right), \\
& L_{2}=\operatorname{diag}\left(l_{1}^{+}, l_{2}^{+}, \ldots, l_{n}^{+}\right) .
\end{aligned}
$$

(H3) The noise matrix $\sigma(t, i, \cdot, \cdot, \cdot, \cdot)$ is local Lipschitz continuous and satisfies the linear growth condition as well, and $\sigma(0, i, 0,0,0,0)=0$. Moreover, there exist positive definite matrices $H_{1 i}, H_{2 i}, H_{3 i}$, and $H_{4 i}(i \in S)$ such that

$$
\begin{array}{r}
\operatorname{trace}\left[\sigma^{T}\left(t, i, z_{1}, z_{2}, z_{3}, z_{4}\right) \sigma\left(t, i, z_{1}, z_{2}, z_{3}, z_{4}\right)\right] \\
\leq z_{1}^{T} H_{1 i} z_{1}+z_{2}^{T} H_{2 i} z_{2}+z_{3}^{T} H_{3 i} z_{3}+z_{4}^{T} H_{4 i} z_{4},
\end{array}
$$


(H4) The time-varying admissible parametric uncertainties $\Delta A_{i}(t), \Delta B_{i}(t), \Delta E_{i}(t), i \in S$, are in terms of

$$
\left[\begin{array}{lll}
\Delta A_{i}(t) & \Delta B_{i}(t) & \Delta E_{i}(t)
\end{array}\right]=Z_{i} F_{i}(t)\left[\begin{array}{lll}
H_{i} & J_{i} & K_{i}
\end{array}\right],
$$

where $Z_{i}, H_{i}, J_{i}$, and $K_{i}$ are known real constant matrices with appropriate dimensions and $F_{i}(t)$ is the uncertain time-varying matrix-valued function satisfying

$$
F_{i}^{T}(t) F_{i}(t) \leq I, \quad \forall t \geq 0 .
$$

In this paper, we always assume that some conditions are satisfied so that system (3) has a unique equilibrium point. Let $u^{*}=\left(u_{1}^{*}, u_{2}^{*}, \ldots, u_{n}^{*}\right) \in \mathbb{R}^{n}$ be the equilibrium point of system (3). For simplicity, we can shift the equilibrium $u^{*}$ to the origin by letting $x(t)=u(t)-u^{*}$. Then system (3) can be transformed into the following one:

$$
\begin{aligned}
& d\left[x(t)-D_{i} x\left(t-\tau_{3}(t)\right)\right]=\left[-C_{i} x(t)+\left(A_{i}+\Delta A_{i}\right)\right. \\
& \cdot g(x(t))+\left(B_{i}+\Delta B_{i}\right) g\left(x\left(t-\tau_{1}(t)\right)\right) \\
& \left.+\left(E_{i}+\Delta E_{i}\right) \int_{t-\tau_{2}(t)}^{t} g(x(s)) d s\right] d t+\sigma(t, i, x(t), \\
& \left.x\left(t-\tau_{1}(t)\right), x\left(t-\tau_{2}(t)\right), x\left(t-\tau_{3}(t)\right)\right) d \omega(t), \\
& \mathscr{D} x\left(t_{k}^{+}\right)=W_{i k} \mathscr{D} x\left(t_{k}^{-}\right), \quad t=t_{k}, k \in t_{k},
\end{aligned}
$$

where $g(x(\cdot))=f\left(u(\cdot)+u^{*}\right)-f\left(u^{*}\right)$. The initial condition of system (10) is given in terms of

$$
\begin{aligned}
x(s) & =\psi(s)=\varphi(s)-u^{*}, \quad s \in[-\tau, 0], \\
r(0) & =i_{0}, \\
\tau & =\max \left\{h_{2}+\tau_{3}, \tau_{2}+\tau_{3}\right\} .
\end{aligned}
$$

Noting that $g(0)=0$ and $\sigma(0, i, 0,0,0,0)=0$, we know that the trivial solution of system (10) exists. Thus, the stability problem of $u^{*}$ of system (3) converts to the stability problem of the trivial solution of system (10). On the other hand, from hypothesis $(H 1)$, we get

$$
l_{j}^{-} \leq \frac{g_{j}(a)-g_{j}(b)}{a-b} \leq l_{j}^{+},
$$

for any $a, b \in \mathbb{R}, a \neq b, j=1,2, \ldots, n$.

Next, let $x(t ; \xi)$ denote the state trajectory from the initial data $x(\theta)=\xi(\theta)$ on $-\tau \leq \theta \leq 0$ in $L_{\mathscr{F}_{t}}^{2}\left([-\tau, 0] ; \mathbb{R}^{n}\right)$. Based on above discussion, system (10) has a trivial solution $x(t ; 0) \equiv 0$ corresponding to the initial condition $\xi=0$. For simplicity, we write $x(t ; \xi)=x(t)$.

The following definition and lemmas are useful for developing our main results.
Definition 2 (see [49]). The trivial solution of system (10) is said to be exponentially stable in mean square if for every $\xi \epsilon$ $L_{\mathscr{F}_{0}}^{2}\left([-\tau, 0] ; \mathbb{R}^{n}\right)$, there exist constants $\gamma>0$ and $\mathscr{M}>0$ such that the following inequality holds:

$$
E\|x(t ; \xi)\|^{2} \leq \mathscr{M} e^{-\gamma t} \sup _{-\tau \leq \theta \leq 0} E\|\xi(\theta)\|^{2}
$$

where $\gamma$ is called the exponential convergence rate.

Lemma 3 (Jensen integral inequality; see $\mathrm{Gu}$ [50]). For any constant matrix $M>0$, any scalars $s_{1}$ and $s_{2}$ with $s_{1}<s_{2}$, and $a$ vector function $\eta(t):[a, b] \rightarrow \mathbb{R}$ such that the integrals concerned are well defined, then the following inequality holds:

$$
\begin{gathered}
\left(\int_{s_{1}}^{s_{2}} \eta(s) d s\right)^{T} M\left(\int_{s_{1}}^{s_{2}} \eta(s) d s\right) \\
\quad \leq\left(s_{2}-s_{1}\right) \int_{s_{1}}^{s_{2}} \eta(s) M \eta(s) d s .
\end{gathered}
$$

Lemma 4 (Wang et al. [51]). For given matrices $E, F$, and $G$ with $F^{T} F \leq I$ and scalar $\varepsilon>0$, the following inequality holds:

$$
G F E+E^{T} F^{T} G^{T} \leq \varepsilon G G^{T}+\varepsilon^{-1} E^{T} E .
$$

Remark 5. Some inequalities have been widely used to derive less conservative conditions to analyze and synthesize problems of time-delay systems, for example, GronwallBellman inequality [52], Halanay inequality [53], Jensen integral inequality, Wirtinger integral [54], and reciprocally convex approach [55] in which Jensen integral inequality is the most used, and Lemma 4 also holds if $s_{1}=s_{2}$.

Remark 6. Similar to [8], we further investigate the substantial influence of the three-type impulses for the exponential stability issue of stochastic neural networks of neutral-type with both Markovian jump parameters and mixed time delays.

\section{Main Results}

In this section, the robust exponential stability in mean square of the trivial solution for system (10) is studied under hypotheses $(H 1)$ to $(H 4)$.

Before proceeding, by using the model transformation technique, we rewritten system (10) as

$$
\begin{array}{r}
d\left[x(t)-D_{i} x\left(t-\tau_{3}(t)\right)\right]=z(t) d t+\sigma(t) d \omega(t), \\
t \neq t_{k}, k \in \mathbb{N}^{+},
\end{array}
$$

where

$$
\begin{aligned}
& z(t)=-C_{i} x(t)+\left(A_{i}+\Delta A_{i}\right) g(x(t))+\left(B_{i}+\Delta B_{i}\right) \\
& \cdot g\left(x\left(t-\tau_{1}(t)\right)\right)+\left(E_{i}+\Delta E_{i}\right) \int_{t-\tau_{2}(t)}^{t} g(x(s)) d s, \\
& \sigma(t)=\sigma\left(t, i, x(t), x\left(t-\tau_{1}(t)\right), x\left(t-\tau_{2}(t)\right),\right. \\
& \left.\quad x\left(t-\tau_{3}(t)\right)\right) .
\end{aligned}
$$


Theorem 7. Assume that hypotheses (H1)-(H4) hold. For given scalars $h_{1}, h_{2}, \tau_{2}, \tau_{3}$, and $\mu_{1}, \mu_{2}, \mu_{3}$, the trivial solution of system (10) is robustly exponentially stable in mean square if there exist positive scalars $\lambda_{i}, \alpha_{i} \geq-1\left(\alpha_{i} \neq 0\right), \alpha=\max \{1+$ $\left.\alpha_{i}\right\}$ ( $\left.i \in S\right), \kappa_{1}, \kappa_{2}, \gamma$, positive definite matrices $P_{i}(i \in S), Q_{1}$, $Q_{2}, Q_{3}, Q_{4}, Q_{5}, Q_{6}$, positive diagonal matrices $R_{i}, S_{i}(i \in S)$, and any real matrices $N_{q}(q=1,2, \ldots, 10)$ of appropriate dimensions such that

$$
\begin{aligned}
P_{i} & \leq \lambda_{i} I, \\
W_{i k}^{T} P_{l} W_{i k}-P_{i} \leq \alpha_{i} P_{i} & {\left[\text { here } r\left(t_{k}\right)=l\right], } \\
\Phi_{i} & =\left[\begin{array}{ccc}
\Phi_{i}^{\prime} & \Gamma_{1 i} & \Gamma_{2 i} \\
* & -\kappa_{1} I & 0 \\
* & * & -\kappa_{2} I
\end{array}\right]<0,
\end{aligned}
$$

where

$$
\begin{aligned}
& \Phi_{i}^{\prime}=\left(\phi_{i m n}^{\prime}\right)_{13 \times 13}, \\
& m=1,2, \ldots, 13, n=1,2, \ldots, 13, \\
& \Gamma_{1 i}=\left[\begin{array}{c}
P_{i} Z_{i} \\
0_{12 n \times n}
\end{array}\right], \\
& \Gamma_{2 i}=\left[\begin{array}{c}
D_{i}^{T} P_{i} Z_{i} \\
0_{12 n \times n}
\end{array}\right] \text {, } \\
& \phi_{i 1,1}^{\prime}=-P_{i} C_{i}-C_{i}^{T} P_{i}+\gamma P_{i}+\lambda_{i} H_{1 i}+\sum_{j=1}^{N} \pi_{i j} P_{j} \\
& +e^{-\gamma h_{2}} Q_{1}+\sum_{j=2}^{3} e^{-\gamma \tau_{j}} Q_{j} \\
& +\frac{h_{2}-h_{1}}{\gamma}\left(e^{\gamma h_{2}}-e^{\gamma h_{1}}\right) Q_{5} \\
& +\frac{\tau_{3}}{\gamma}\left(e^{\gamma \tau_{3}}-1\right) Q_{6}-2 L_{1} R_{i} L_{2}+N_{1}+N_{1}^{T} \\
& +N_{6}+N_{6}^{T} \\
& \phi_{i 1,2}^{\prime}=-N_{1}+N_{2}^{T} \text {, } \\
& \phi_{i 1,3}^{\prime}=-N_{6}+N_{7}^{T} \text {, } \\
& \phi_{i 1,4}^{\prime}=-\left(\sum_{j}^{N} \pi_{i j} P_{j}\right) D_{i}-\gamma P_{i} D_{i}+C_{i}^{T} P_{i} D_{i}-N_{1} D_{i} \\
& -N_{6} D_{i}+N_{3}^{T}+N_{8}^{T}, \\
& \phi_{i 1,5}^{\prime}=N_{1} D_{i}+N_{4}^{T}, \\
& \phi_{i 1,6}^{\prime}=N_{6} D_{i}+N_{9}^{T},
\end{aligned}
$$

$$
\begin{aligned}
\phi_{i 1,7}^{\prime}= & P_{i} A_{i}+\left(L_{1}+L_{2}\right) R_{i}, \\
\phi_{i 1,8}^{\prime}= & P_{i} B_{i}, \\
\phi_{i 1,9}^{\prime}= & P_{i} E_{i}, \\
\phi_{i 1,10}^{\prime}= & -N_{1}+N_{5}^{T}, \\
\phi_{i 1,11}^{\prime}= & -N_{6}+N_{10}^{T}, \\
\phi_{i 2,2}^{\prime}= & \lambda_{i} H_{2 i}-\left(1-\mu_{1}\right) h\left(\mu_{1}\right) Q_{1}-2 L_{1} S_{i} L_{2}-N_{2} \\
& -N_{2}^{T}, \\
\phi_{i 2,4}^{\prime}= & -N_{2} D_{i}-N_{3}^{T}, \\
\phi_{i 2,5}^{\prime}= & N_{2} D_{i}-N_{4}^{T}, \\
\phi_{i 2,8}^{\prime}= & \left(L_{1}+L_{2}\right) S_{i},
\end{aligned}
$$$$
\phi_{i 2,10}^{\prime}=-N_{2}-N_{5}^{T} \text {, }
$$$$
\phi_{i 3,3}^{\prime}=\lambda_{i} H_{3 i}-\left(1-\mu_{2}\right) h\left(\mu_{2}\right) Q_{2}-N_{7}-N_{7}^{T},
$$$$
\phi_{i 3,4}^{\prime}=-N_{7} D_{i}-N_{8}^{T} \text {, }
$$$$
\phi_{i 3,6}^{\prime}=N_{7} D_{i}-N_{9}^{T} \text {, }
$$$$
\phi_{i 3,11}^{\prime}=-N_{7}-N_{10}^{T} \text {, }
$$$$
\phi_{i 4,4}^{\prime}=\gamma D_{i}^{T} P_{i} D_{i}+D_{i}^{T}\left(\sum_{j=1}^{N} \pi_{i j} P_{j}\right) D_{i}+\lambda_{i} H_{4 i}
$$$$
-\left(1-\mu_{3}\right) h\left(\mu_{3}\right) Q_{3}-N_{3} D_{i}-D_{i}^{T} N_{3}^{T}
$$$$
-N_{8} D_{i}-D_{i}^{T} N_{8}^{T}
$$$$
\phi_{i 4,5}^{\prime}=N_{3} D_{i}-D_{i}^{T} N_{4}^{T} \text {, }
$$$$
\phi_{i 4,6}^{\prime}=N_{8} D_{i}-D_{i}^{T} N_{9}^{T},
$$$$
\phi_{i 4,7}^{\prime}=-D_{i}^{T} P_{i} A_{i},
$$$$
\phi_{i 4,8}^{\prime}=-D_{i}^{T} P_{i} B_{i},
$$$$
\phi_{i 4,9}^{\prime}=-D_{i}^{T} P_{i} E_{i},
$$$$
\phi_{i 4,10}^{\prime}=-N_{3}-D_{i}^{T} N_{5}^{T} \text {, }
$$$$
\phi_{i 4,11}^{\prime}=-N_{8}-D_{i}^{T} N_{10}^{T} \text {, }
$$$$
\phi_{i 5,5}^{\prime}=N_{4} D_{i}+D_{i}^{T} N_{4}^{T} \text {, }
$$$$
\phi_{5,10}^{\prime}=-N_{4}+D_{i}^{T} N_{5}^{T},
$$$$
\phi_{i 6,6}^{\prime}=N_{9} D_{i}+D_{i}^{T} N_{9}^{T} \text {, }
$$$$
\phi_{i 6,11}^{\prime}=-N_{9}+D_{i}^{T} N_{10}^{T} \text {, }
$$$$
\phi_{i 7,7}^{\prime}=\frac{\tau}{\gamma}\left(e^{\gamma \tau}-1\right) Q_{4}-2 R_{i}+\kappa_{1} H_{i}^{T} H_{i}+\kappa_{2} H_{i}^{T} H_{i},
$$ 


$$
\begin{aligned}
\phi_{i 8,8}^{\prime} & =-2 S_{i}+\kappa_{1} J_{i}^{T} J_{i}+\kappa_{2} J_{i}^{T} J_{i}, \\
\phi_{i 9,9}^{\prime} & =-Q_{4}+\kappa_{1} K_{i}^{T} K_{i}+\kappa_{2} K_{i}^{T} K_{i}, \\
\phi_{i 10,10}^{\prime} & =-N_{5}-N_{5}^{T}, \\
\phi_{i 11,11}^{\prime} & =-N_{10}-N_{10}^{T}, \\
\phi_{i 12,12}^{\prime} & =-Q_{5}, \\
\phi_{i 13,13}^{\prime} & =-Q_{6},
\end{aligned}
$$

and the function $h(u) \in \mathbb{R}^{+}, u \in \mathbb{R}$, is defined as

$$
h(u)= \begin{cases}1, & u>1, \\ e^{-2 \gamma \tau}, & u \leq 1\end{cases}
$$

and for $\alpha_{i}>0,-\gamma+\ln \alpha / \inf \left\{t_{k}-t_{k-1}\right\}<0, k \in \mathbb{N}^{+}$, other elements of $\Phi_{i}^{\prime}$ are all equal to 0 .

Proof. Let $x_{t}=x(t+s), s \in[-\tau, 0]$. As discussed in [56-59], $\left\{x_{t}, r(t), t \geq 0\right\}$ is a $C\left([-\tau, 0] ; \mathbb{R}^{n}\right) \times S$-valued Markov process. Construct the following stochastic Lyapunov-Krasovskii functional candidate for system (10):

$$
V\left(t, i, x_{t}\right)=V_{1}\left(t, i, x_{t}\right)+V_{2}\left(t, i, x_{t}\right)+V_{3}\left(t, i, x_{t}\right),
$$

where

$$
\begin{aligned}
& V_{1}\left(t, i, x_{t}\right)=e^{\gamma t}\left(x(t)-D_{i} x\left(t-\tau_{3}(t)\right)\right)^{T} \\
& \cdot P_{i}\left(x(t)-D_{i} x\left(t-\tau_{3}(t)\right)\right), \\
& V_{2}\left(t, i, x_{t}\right)=\int_{t-\tau_{1}(t)}^{t} e^{\gamma\left(s-h_{2}\right)} x^{T}(s) Q_{1} x(s) d s \\
& \quad+\int_{t-\tau_{2}(t)}^{t} e^{\gamma\left(s-\tau_{2}\right)} x^{T}(s) Q_{2} x(s) d s \\
& \quad+\int_{t-\tau_{3}(t)}^{t} e^{\gamma\left(s-\tau_{3}\right)} x^{T}(s) Q_{3} x(s) d s, \\
& V_{3}\left(t, i, x_{t}\right) \\
& \quad=\tau \int_{-\tau}^{0} \int_{t+\beta}^{t} e^{\gamma(s-\beta)} g^{T}(x(s)) Q_{4} g(x(s)) d s d \beta \\
& \quad+\left(h_{2}-h_{1}\right) \int_{-h_{2}}^{-h_{1}} \int_{t+\beta}^{t} e^{\gamma(s-\beta)} x^{T}(s) Q_{5} x(s) d s d \beta \\
& +\tau_{3} \int_{-\tau_{3}}^{0} \int_{t+\beta}^{t} e^{\gamma(s-\beta)} x^{T}(s) Q_{6} x(s) d s d \beta .
\end{aligned}
$$

For $t \in\left[t_{k-1}, t_{k}\right), k \in \mathbb{N}^{+}$, denote $\mathbb{\Omega}$ to be the weak infinitesimal operator of the random process $x_{t}=x(t+s)$, $s \in[-\tau, 0]$; then along the trajectory of system (10) we have

$$
\begin{aligned}
\mathfrak{Q} V\left(t, i, x_{t}\right)= & \mathfrak{Q} V_{1}\left(t, i, x_{t}\right)+\mathfrak{Q} V_{2}\left(t, i, x_{t}\right) \\
& +\mathfrak{Q} V_{3}\left(t, i, x_{t}\right),
\end{aligned}
$$

where

$$
\begin{aligned}
& \mathfrak{Q} V_{1}\left(t, i, x_{t}\right)=\gamma e^{\gamma t}\left(x(t)-D_{i} x\left(t-\tau_{3}(t)\right)\right)^{T} P_{i}(x(t) \\
& \left.-D_{i} x\left(t-\tau_{3}(t)\right)\right)+2 e^{\gamma t}(x(t) \\
& \left.-D_{i} x\left(t-\tau_{3}(t)\right)\right)^{T} P_{i}\left[-C_{i} x(t)\right. \\
& +\left(A_{i}+\Delta A_{i}\right) g(x(t)) \\
& +\left(B_{i}+\Delta B_{i}\right) g\left(x\left(t-\tau_{1}(t)\right)\right) \\
& \left.+\left(E_{i}+\Delta E_{i}\right) \int_{t-\tau_{2}(t)}^{t} g(x(s)) d s\right] \\
& +e^{\gamma t} \operatorname{trace}\left[\sigma^{T}(t) P_{i} \sigma(t)\right]+e^{\gamma t}(x(t) \\
& \left.-D_{i} x\left(t-\tau_{3}(t)\right)\right)^{T}\left(\sum_{j=1}^{N} \pi_{i j} P_{j}\right)(x(t) \\
& \left.-D_{i} x\left(t-\tau_{3}(t)\right)\right) \\
& \mathfrak{Q} V_{2}\left(t, i, x_{t}\right)=e^{\gamma\left(t-h_{2}\right)} x^{T}(t) Q_{1} x(t)-\left(1-\dot{\tau}_{1}(t)\right) \\
& \cdot e^{\gamma\left(t-\tau_{1}(t)-h_{2}\right)} x^{T}\left(t-\tau_{1}(t)\right) Q_{1} x\left(t-\tau_{1}(t)\right) \\
& +e^{\gamma\left(t-\tau_{2}\right)} x^{T}(t) Q_{2} x(t)-\left(1-\dot{\tau}_{2}(t)\right) \\
& \cdot e^{\gamma\left(t-\tau_{2}(t)-\tau_{2}\right)} x^{T}\left(t-\tau_{2}(t)\right) Q_{2} x\left(t-\tau_{2}(t)\right) \\
& +e^{\gamma\left(t-\tau_{3}\right)} x^{T}(t) Q_{3} x(t)-\left(1-\dot{\tau}_{3}(t)\right) \\
& \text { - } e^{\gamma\left(t-\tau_{3}(t)-\tau_{3}\right)} x^{T}\left(t-\tau_{3}(t)\right) Q_{3} x\left(t-\tau_{3}(t)\right), \\
& \mathcal{Q} V_{3}\left(t, i, x_{t}\right)=\tau \int_{-\tau}^{0} e^{\gamma(t-\beta)} g^{T}(x(t)) Q_{4} g(x(t)) d \beta \\
& -\tau \int_{-\tau}^{0} e^{\gamma t} g^{T}(x(t+\beta)) Q_{4} g(x(t+\beta)) d \beta \\
& +\left(h_{2}-h_{1}\right) \int_{-h_{2}}^{-h_{1}} e^{\gamma(t-\beta)} x^{T}(t) Q_{5} x(t) d \beta-\left(h_{2}\right. \\
& \left.-h_{1}\right) \int_{-h_{2}}^{-h_{1}} e^{\gamma t} x^{T}(t+\beta) Q_{5} x(t+\beta) d \beta \\
& +\tau_{3} \int_{-\tau_{3}}^{0} e^{\gamma(t-\beta)} x^{T}(t) Q_{6} x(t) d \beta \\
& -\tau_{3} \int_{-\tau_{3}}^{0} e^{\gamma t} x^{T}(t+\beta) Q_{6} x(t+\beta) d \beta=\frac{\tau}{\gamma}\left(e^{\gamma \tau}\right. \\
& \text { - 1) } e^{\gamma t} g^{T}(x(t)) Q_{4} g(x(t)) \\
& -\tau e^{\gamma t} \int_{t-\tau}^{t} g^{T}(x(s)) Q_{4} g(x(s)) d s \\
& +\frac{h_{2}-h_{1}}{\gamma}\left(e^{\gamma h_{2}}-e^{\gamma h_{1}}\right) e^{\gamma t} x^{T}(t) Q_{5} x(t)-\left(h_{2}\right.
\end{aligned}
$$




$$
\begin{aligned}
& \left.-h_{1}\right) e^{\gamma t} \int_{t-h_{2}}^{t-h_{1}} x^{T}(s) Q_{5} x(s) d s+\frac{\tau_{3}}{\gamma}\left(e^{\gamma \tau_{3}}-1\right) \\
& \cdot e^{\gamma t} x^{T}(t) Q_{6} x(t)-\tau_{3} e^{\gamma t} \int_{t-\tau_{3}}^{t} x^{T}(s) Q_{6} x(s) d s \\
& \leq \frac{\tau}{\gamma}\left(e^{\gamma \tau}-1\right) e^{\gamma t} g^{T}(x(t)) Q_{4} g(x(t))-\tau_{2}(t) \\
& \cdot e^{\gamma t} \int_{t-\tau_{2}(t)}^{t} g^{T}(x(s)) Q_{4} g(x(s)) d s \\
& +\frac{h_{2}-h_{1}}{\gamma}\left(e^{\gamma h_{2}}-e^{\gamma h_{1}}\right) e^{\gamma t} x^{T}(t) Q_{5} x(t)-\left(h_{2}\right. \\
& \left.-h_{1}\right) e^{\gamma t} \int_{t-h_{2}}^{t-h_{1}} x^{T}(s) Q_{5} x(s) d s+\frac{\tau_{3}}{\gamma}\left(e^{\gamma \tau_{3}}-1\right) \\
& \cdot e^{\gamma t} x^{T}(t) Q_{6} x(t)-\tau_{3} e^{\gamma t} \int_{t-\tau_{3}}^{t} x^{T}(s) Q_{6} x(s) d s .
\end{aligned}
$$

From hypotheses (H3) and (18), we have

$$
\begin{aligned}
& \operatorname{trace}\left[\sigma^{T}(t) P_{i} \sigma(t)\right] \leq \lambda_{i} \operatorname{trace}\left[\sigma^{T}(t) \sigma(t)\right] \\
& \quad \leq \lambda_{i}\left(x^{T}(t) H_{1 i} x(t)\right. \\
& +x^{T}\left(t-\tau_{1}(t)\right) H_{2 i} x\left(t-\tau_{1}(t)\right) \\
& +x^{T}\left(t-\tau_{2}(t)\right) H_{3 i} x\left(t-\tau_{2}(t)\right) \\
& \left.+x^{T}\left(t-\tau_{3}(t)\right) H_{4 i} x\left(t-\tau_{3}(t)\right)\right) .
\end{aligned}
$$

Combining (20) and (27) together yields

$$
\begin{aligned}
\mathfrak{S} V_{2} & \left(t, i, x_{t}\right) \\
& \leq e^{\gamma t}\left(x^{T}(t)\left(e^{-\gamma h_{2}} Q_{1}+e^{-\gamma \tau_{2}} Q_{2}+e^{-\gamma \tau_{3}} Q_{3}\right) x(t)\right. \\
& -\left(1-\mu_{1}\right) h\left(\mu_{1}\right) x^{T}\left(t-\tau_{1}(t)\right) Q_{1} x\left(t-\tau_{1}(t)\right) \\
& -\left(1-\mu_{2}\right) h\left(\mu_{2}\right) x^{T}\left(t-\tau_{2}(t)\right) Q_{2} x\left(t-\tau_{2}(t)\right) \\
& \left.-\left(1-\mu_{3}\right) h\left(\mu_{3}\right) x^{T}\left(t-\tau_{3}(t)\right) Q_{3} x\left(t-\tau_{3}(t)\right)\right) .
\end{aligned}
$$

If $\tau_{2}(t)>0, h_{2}>h_{1}$, based on (28) and Lemma 3 , it is easy to derive that

$$
\begin{aligned}
\mathcal{L} V_{3}\left(t, i, x_{t}\right) \leq & \frac{\tau}{\gamma}\left(e^{\gamma \tau}-1\right) e^{\gamma t} g^{T}(x(t)) Q_{4} g(x(t)) \\
& -e^{\gamma t}\left[\int_{t-\tau_{2}(t)}^{t} g(x(s)) d s\right]^{T}
\end{aligned}
$$

$$
\begin{aligned}
& \cdot Q_{4}\left[\int_{t-\tau_{2}(t)}^{t} g(x(s)) d s\right] \\
& +\frac{h_{2}-h_{1}}{\gamma}\left(e^{\gamma h_{2}}-e^{\gamma h_{1}}\right) e^{\gamma t} x^{T}(t) \\
& \cdot Q_{5} x(t)-e^{\gamma t}\left[\int_{t-h_{2}}^{t-h_{1}} x(s) d s\right]^{T} \\
& \cdot Q_{5}\left[\int_{t-h_{2}}^{t-h_{1}} x(s) d s\right]+\frac{\tau_{3}}{\gamma}\left(e^{\gamma \tau_{3}}-1\right) \\
& \cdot e^{\gamma t} x^{T}(t) Q_{6} x(t) \\
& -e^{\gamma t}\left[\int_{t-\tau_{3}}^{t} x(s) d s\right]^{T} \\
& \cdot Q_{6}\left[\int_{t-\tau_{3}}^{t} x(s) d s\right] .
\end{aligned}
$$

Note that inequality (31) still holds if $\tau_{2}(t)=0$ and $h_{2}=h_{1}$ since

$$
\begin{aligned}
& \int_{t-\tau_{2}(t)}^{t} g^{T}(x(s)) Q_{4} g(x(s)) d s \\
& =\left[\int_{t-\tau_{2}(t)}^{t} g(x(s)) d s\right]^{T} Q_{4}\left[\int_{t-\tau_{2}(t)}^{t} g(x(s)) d s\right] \\
& =0 \\
& \int_{t-h_{2}}^{t-h_{1}} x^{T}(s) Q_{5} x(s) d s\left[\int_{t-h_{2}}^{t-h_{1}} x(s) d s\right]^{T} \\
& \cdot Q_{5}\left[\int_{t-h_{2}}^{t-h_{1}} x(s) d s\right]=0, \\
& \int_{t-\tau_{3}}^{t} x^{T}(s) Q_{6} x(s) d s=\left[\int_{t-\tau_{3}}^{t} x(s) d s\right]^{T} \\
& \cdot Q_{6}\left[\int_{t-\tau_{3}}^{t} x(s) d s\right]=0 .
\end{aligned}
$$

On the other hand, by hypothesis $(\mathrm{H} 2)$, one can get that there exist positive diagonal matrices $R_{i}=\operatorname{diag}\left\{r_{1 i}, r_{2 i}, \ldots\right.$, $\left.r_{n i}\right\}, S_{i}=\operatorname{diag}\left\{s_{1 i}, s_{2 i}, \ldots, s_{n i}\right\}, i \in S$, such that the following inequalities hold

$$
\begin{aligned}
0 & \leq 2 e^{\gamma t} \sum_{j=1}^{n} r_{j i}\left(g_{j}\left(x_{j}(t)\right)-l_{j}^{-} x_{j}(t)\right)\left(l_{j}^{+} x_{j}(t)\right. \\
& \left.-g_{j}\left(x_{j}(t)\right)\right)=2 e^{\gamma t}\left(x^{T}(t)\left(L_{1}+L_{2}\right) R_{i} g(x(t))\right. \\
& \left.-x^{T}(t) L_{1} R_{i} L_{2} x(t)-g^{T}(x(t)) R_{i} g(x(t))\right),
\end{aligned}
$$




$$
\begin{aligned}
0 \leq & 2 e^{\gamma t} \sum_{j=1}^{n} s_{j i}\left(g_{j}\left(x_{j}\left(t-\tau_{1}(t)\right)\right)-l_{j}^{-} x_{j}\left(t-\tau_{1}(t)\right)\right) \\
& \cdot\left(l_{j}^{+} x_{j}\left(t-\tau_{1}(t)\right)-g_{j}\left(x_{j}\left(t-\tau_{1}(t)\right)\right)\right) \\
& =2 e^{\gamma t}\left(x^{T}\left(t-\tau_{1}(t)\right)\left(L_{1}+L_{2}\right) S_{i} g\left(x\left(t-\tau_{1}(t)\right)\right)\right. \\
& -x^{T}\left(t-\tau_{1}(t)\right) L_{1} S_{i} L_{2} x\left(t-\tau_{1}(t)\right) \\
& \left.-g^{T}\left(x\left(t-\tau_{1}(t)\right)\right) S_{i} g\left(x\left(t-\tau_{1}(t)\right)\right)\right) .
\end{aligned}
$$

Moreover, by utilizing the well-known Newton-Leibniz formulae and (16), it can be deduced that for any matrices $N_{q}$, $q=1,2, \ldots, 10$, with appropriate dimensions, the following equalities also hold

$$
\begin{aligned}
0 & =2 e^{\gamma t}\left[x^{T}(t) N_{1}+x^{T}\left(t-\tau_{1}(t)\right) N_{2}\right. \\
& +x^{T}\left(t-\tau_{3}(t)\right) N_{3} \\
& +x^{T}\left(t-\tau_{1}(t)-\tau_{3}\left(t-\tau_{1}(t)\right)\right) N_{4} \\
& \left.+\left(\int_{t-\tau_{1}(t)}^{t} z(s) d s\right)^{T} N_{5}\right] \\
& +\left[\left(x(t)-D_{i} x\left(t-\tau_{3}(t)\right)\right)\right. \\
& -\left(x\left(t-\tau_{1}(t)\right)-D_{i} x\left(t-\tau_{1}(t)-\tau_{3}\left(t-\tau_{1}(t)\right)\right)\right) \\
& \left.-\int_{t-\tau_{1}(t)}^{t} z(s) d s-\int_{t-\tau_{1}(t)}^{t} \sigma(s) d \omega(s)\right] \\
& \left.+\int_{t-\tau_{2}(t)}^{t} z(s) d s-\int_{t-\tau_{2}(t)}^{t} \sigma(s) d \omega(s)\right] \\
& +\left(x(t)-x_{i}^{T}(t) N_{6}+x^{T}\left(t-\tau_{2}(t)\right) N_{7}\right. \\
& +x^{T}\left(t-\tau_{3}(t)\right) N_{8} \\
& +x^{T}\left(t-\tau_{2}(t)-\tau_{3}\left(t-\tau_{2}(t)\right)\right) N_{9} \\
& \left.+(s) d s)^{T} N_{10}\right]
\end{aligned}
$$

Considering hypothesis (H4), substituting (26)-(34) and $E d \omega(t)=0$ into (25) yields that for $t \in\left[t_{k-1}, t_{k}\right), k \in \mathbb{N}^{+}$,

$$
E \mathfrak{Q} V\left(t, i, x_{t}\right) \leq e^{\gamma t} E \chi^{T}(t) \Phi_{i}^{\prime \prime} \chi(t)
$$

where

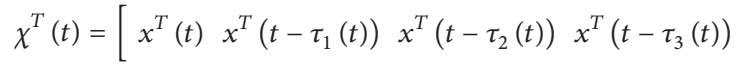

$$
\begin{aligned}
& x^{T}\left(t-\tau_{1}(t)-\tau_{3}\left(t-\tau_{1}(t)\right)\right) \\
& x^{T}\left(t-\tau_{2}(t)-\tau_{3}\left(t-\tau_{2}(t)\right)\right) g^{T}(x(t)) \\
& g^{T}\left(x\left(t-\tau_{1}(t)\right)\right)\left(\int_{t-\tau_{2}(t)}^{t} g(x(s)) d s\right)^{T} \\
& \left(\int_{t-\tau_{1}(t)}^{t} z(s) d s\right)^{T}\left(\int_{t-\tau_{2}(t)}^{t} z(s) d s\right)^{T} \\
& \left.\left(\int_{t-h_{2}}^{t-h_{1}} x(s) d s\right)^{T}\left(\int_{t-\tau_{3}}^{t} x(s) d s\right)^{T}\right], \\
& \Phi_{i}^{\prime \prime}=\left.\Phi_{i}^{\prime}\right|_{\kappa_{1}=0, \kappa_{2}=0}+\left[\begin{array}{c}
P_{i} Z_{i} \\
0_{12 n \times n}
\end{array}\right] F_{i}(t)\left[\begin{array}{c}
0_{6 n \times n} \\
H_{i}^{T} \\
J_{i}^{T} \\
K_{i}^{T} \\
0_{4 n \times n}
\end{array}\right]^{T} \\
& +\left[\begin{array}{c}
0_{6 n \times n} \\
H_{i}^{T} \\
J_{i}^{T} \\
K_{i}^{T} \\
0_{4 n \times n}
\end{array}\right] F_{i}^{T}(t)\left[\begin{array}{c}
P_{i} Z_{i} \\
0_{12 n \times n}
\end{array}\right]^{T} \\
& -\left[\begin{array}{c}
D_{i}^{T} P_{i} Z_{i} \\
0_{12 n \times n}
\end{array}\right] F_{i}(t)\left[\begin{array}{c}
0_{6 n \times n} \\
H_{i}^{T} \\
J_{i}^{T} \\
K_{i}^{T} \\
0_{4 n \times n}
\end{array}\right]^{T} \\
& -\left[\begin{array}{c}
0_{6 n \times n} \\
H_{i}^{T} \\
J_{i}^{T} \\
K_{i}^{T} \\
0_{4 n \times n}
\end{array}\right] F_{i}^{T}(t)\left[\begin{array}{c}
D_{i}^{T} P_{i} Z_{i} \\
0_{12 n \times n}
\end{array}\right]^{T} \text {. }
\end{aligned}
$$

Combining Lemma 4 and (35) together yields that there exist two positive scalars $\kappa_{1}$ and $\kappa_{2}$ such that

$$
\begin{aligned}
\Phi_{i}^{\prime \prime} \leq & \Xi_{i} \\
= & \left.\Phi_{i}^{\prime}\right|_{\kappa_{1}>0, \kappa_{2}>0}+\kappa_{1}^{-1}\left[\begin{array}{c}
P_{i} Z_{i} \\
0_{12 n \times n}
\end{array}\right]\left[\begin{array}{c}
P_{i} Z_{i} \\
0_{12 n \times n}
\end{array}\right]^{T} \\
& +\kappa_{2}^{-1}\left[\begin{array}{c}
D_{i}^{T} P_{i} Z_{i} \\
0_{12 n \times n}
\end{array}\right]\left[\begin{array}{c}
D_{i}^{T} P_{i} Z_{i} \\
0_{12 n \times n}
\end{array}\right]^{T} .
\end{aligned}
$$

Applying the Schur complement equivalence [60] to (20) yields $\Xi_{i}<0$. Therefore, $\Phi_{i}^{\prime \prime}<0$, which means

$$
E \mathfrak{Q V}\left(t, i, x_{t}\right) \leq 0, \quad t \in\left[t_{k-1}, t_{k}\right), k \in \mathbb{N}^{+} .
$$


For $t=t_{k}, k \in \mathbb{N}^{+}$, according to (19) and (23) and $E d \omega(t)=0$, we have

$$
\begin{aligned}
E V\left(t_{k}, l, x_{t_{k}}\right) & \\
= & E V\left(t_{k}^{-}, i, x_{t_{k}^{-}}\right) \\
& +E e^{\gamma t_{k}} \mathscr{D} x^{T}\left(t_{k}^{-}\right)\left(W_{i k}^{T} P_{l} W_{i k}-P_{i}\right) \mathscr{D} x\left(t_{k}^{-}\right) \\
\leq & E V\left(t_{k}^{-}, i, x_{t_{k}^{-}}\right)+\alpha_{i} E V_{1}\left(t_{k}^{-}, i, x_{t_{k}^{-}}\right) ;
\end{aligned}
$$

if $-1 \leq \alpha_{i}<0$, then

$$
E V\left(t_{k}, l, x_{t_{k}}\right) \leq E V\left(t_{k}^{-}, i, x_{t_{k}^{-}}\right)
$$

if $\alpha_{i}>0$, then

$$
\begin{aligned}
E V\left(t_{k}, l, x_{t_{k}}\right) & \leq\left(1+\alpha_{i}\right) E V\left(t_{k}^{-}, i, x_{t_{k}^{-}}\right) \\
& \leq \alpha E V\left(t_{k}^{-}, i, x_{t_{k}^{-}}\right) .
\end{aligned}
$$

So, from inequalities (38) and (40), for all $i \in S, t \geq 0$, it is true through the mathematical induction that

$$
E V\left(t, i, x_{t}\right) \leq E V\left(0, r(0), x_{0}\right), \quad-1 \leq \alpha_{i}<0 .
$$

Similarly, based on inequalities (38) and (41), for all $i \in S$, $t \in\left[t_{k-1}, t_{k}\right), k \in \mathbb{N}^{+}$, it is true through the mathematical induction that

$$
\begin{aligned}
E V\left(t, i, x_{t}\right) & \leq \alpha^{k-1} E V\left(0, r(0), x_{0}\right) \\
& =E V\left(0, r(0), x_{0}\right) e^{(k-1) \ln \alpha} \\
& \leq E V\left(0, r(0), x_{0}\right) e^{\left(t_{k-1} / \inf \left\{t_{k}-t_{k-1}\right\}\right) \ln \alpha} \\
& \leq E V\left(0, r(0), x_{0}\right) e^{\left(\ln \alpha / \inf \left\{t_{k}-t_{k-1}\right\}\right) t}, \\
& \quad \alpha_{i}>0 .
\end{aligned}
$$

From (23), (42), and (43), the following inequalities are, namely, hold

$$
\begin{aligned}
& E(\mathscr{D} x(t))^{T}(\mathscr{D} x(t)) \leq \frac{E V\left(0, r(0), x_{0}\right)}{\min _{i \in S} \lambda_{\min }\left(P_{i}\right)} e^{-\gamma t}, \\
& -1 \leq \alpha_{i}<0, t \geq 0, \\
& E(\mathscr{D} x(t))^{T}(\mathscr{D} x(t)) \\
& \leq \frac{E V\left(0, r(0), x_{0}\right)}{\min _{i \in S} \lambda_{\min }\left(P_{i}\right)} e^{\left(-\gamma+\ln \alpha / \inf \left\{t_{k}-t_{k-1}\right\}\right) t}, \\
& \alpha_{i}>0, t \in\left[t_{k-1}, t_{k}\right), k \in \mathbb{N}^{+} .
\end{aligned}
$$

On the other hand, defined $L=\operatorname{diag}\left\{l_{1}, l_{2}, \ldots, l_{n}\right\}$ within $l_{j}=$ $\max \left\{\left|l_{j}^{-}\right|,\left|l_{j}^{+}\right|\right\}, j=1,2, \ldots, n$, from Lemma 4 ; it is easy to obtain that there exists a positive scalar $\epsilon$ such that

$$
\begin{aligned}
& E V\left(0, r(0), x_{0}\right)=E\left(x(0)-D_{i} x\left(0-\tau_{3}(0)\right)\right)^{T} \\
& \text { - } P_{r(0)}\left(x(0)-D_{i} x\left(0-\tau_{3}(0)\right)\right) \\
& +\int_{-\tau_{1}(0)}^{0} E e^{\gamma\left(s-h_{2}\right)} x^{T}(s) Q_{1} x(s) d s \\
& +\sum_{j=2}^{3} \int_{-\tau_{j}(0)}^{0} E e^{\gamma\left(s-\tau_{j}\right)} x^{T}(s) Q_{j} x(s) d s \\
& +\tau \int_{-\tau}^{0} \int_{\beta}^{0} E e^{\gamma(s-\beta)} g^{T}(x(s)) Q_{4} g(x(s)) d s d \beta \\
& +\left(h_{2}-h_{1}\right) \int_{-h_{2}}^{-h_{1}} \int_{\beta}^{0} E e^{\gamma(s-\beta)} x^{T}(s) Q_{5} x(s) d s d \beta \\
& +\tau_{3} \int_{-\tau_{3}}^{0} \int_{\beta}^{0} E e^{\gamma(s-\beta)} x^{T}(s) Q_{6} x(s) d s d \beta \leq(1 \\
& +\epsilon) x^{T}(0) P_{r(0)} x(0)+\left(1+\epsilon^{-1}\right) x^{T}\left(-\tau_{3}(0)\right) \\
& \cdot D_{i}^{T} P_{r(0)} D_{i} x\left(-\tau_{3}(0)\right) \\
& +\left(\lambda_{\max }\left(Q_{1}\right) e^{-\gamma h_{2}} \int_{-h_{2}}^{0} e^{\gamma s} d s\right. \\
& +\sum_{j=2}^{3} \lambda_{\max }\left(Q_{j}\right) e^{-\gamma \tau_{j}} \int_{-\tau_{j}}^{0} e^{\gamma s} d s \\
& +\tau \lambda_{\max }\left(L^{T} Q_{4} L\right) \int_{-\tau}^{0} \int_{\beta}^{0} e^{\gamma(s-\beta)} d s d \beta \\
& +\left(h_{2}-h_{1}\right) \lambda_{\max }\left(Q_{5}\right) \int_{-h_{2}}^{-h_{1}} \int_{\beta}^{0} e^{\gamma(s-\beta)} d s d \beta \\
& \left.+\tau_{3} \lambda_{\max }\left(Q_{6}\right) \int_{-\tau_{3}}^{0} \int_{\beta}^{0} e^{\gamma(s-\beta)} d s d \beta\right) \\
& \text {. } \sup _{-\tau \leq \theta \leq 0} E\|\xi(\theta)\|^{2} \\
& \leq\left\{\left[(1+\epsilon)+\left(1+\epsilon^{-1}\right)\left\|D_{i}^{T}\right\|\left\|D_{i}\right\|\right] \max _{i \in S} \lambda_{i}\right. \\
& +\lambda_{\max }\left(Q_{1}\right) \frac{e^{-\gamma h_{2}}}{\gamma}\left(1-e^{-\gamma h_{2}}\right) \\
& +\sum_{j=2}^{3} \lambda_{\max }\left(Q_{j}\right) \frac{e^{-\gamma \tau_{j}}}{\gamma}\left(1-e^{-\gamma \tau_{j}}\right) \\
& +\frac{\tau \lambda_{\max }\left(L^{T} Q_{4} L\right)}{\gamma}\left(\frac{e^{\gamma \tau}-1}{\gamma}-\tau\right)
\end{aligned}
$$




$$
\begin{aligned}
& +\frac{\left(h_{2}-h_{1}\right) \lambda_{\max }\left(Q_{5}\right)}{\gamma}\left(\frac{e^{\gamma h_{2}}-e^{\gamma h_{1}}}{\gamma}-\left(h_{2}-h_{1}\right)\right) \\
& \left.+\frac{\tau_{3} \lambda_{\max }\left(Q_{6}\right)}{\gamma}\left(\frac{e^{\gamma \tau_{3}}-1}{\gamma}-\tau_{3}\right)\right\} \sup _{-\tau \leq \theta \leq 0} E\|\xi(\theta)\|^{2} \\
& =\mathscr{M}_{1} \sup _{-\tau \leq \theta \leq 0} E\|\xi(\theta)\|^{2},
\end{aligned}
$$

where

$$
\begin{aligned}
\mathscr{M}_{1} & {\left[(1+\epsilon)+\left(1+\epsilon^{-1}\right)\left\|D_{i}^{T}\right\|\left\|D_{i}\right\|\right] \max _{i \in S} \lambda_{i} } \\
& +\lambda_{\max }\left(Q_{1}\right) \frac{e^{-\gamma h_{2}}}{\gamma}\left(1-e^{-\gamma h_{2}}\right) \\
& +\sum_{j=2}^{3} \lambda_{\max }\left(Q_{j}\right) \frac{e^{-\gamma \tau_{j}}}{\gamma}\left(1-e^{-\gamma \tau_{j}}\right) \\
& +\frac{\tau \lambda_{\max }\left(L^{T} Q_{4} L\right)}{\gamma}\left(\frac{e^{\gamma \tau}-1}{\gamma}-\tau\right) \\
& +\frac{\left(h_{2}-h_{1}\right) \lambda_{\max }\left(Q_{5}\right)}{\gamma}\left(\frac{e^{\gamma h_{2}}-e^{\gamma h_{1}}}{\gamma}-\left(h_{2}-h_{1}\right)\right) \\
& +\frac{\tau_{3} \lambda_{\max }\left(Q_{6}\right)}{\gamma}\left(\frac{e^{\gamma \tau_{3}}-1}{\gamma}-\tau_{3}\right) .
\end{aligned}
$$

In addition, one can see that

$$
\begin{aligned}
& E x^{T}(t) x(t)=E\left\{\left(\mathscr{D} x(t)+D_{i} x\left(t-\tau_{3}(t)\right)\right)^{T}\right. \\
& \left.\cdot\left(\mathscr{D} x(t)+D_{i} x\left(t-\tau_{3}(t)\right)\right)\right\}=E\left\{(\mathscr{D} x(t))^{T}\right. \\
& \cdot(\mathscr{D} x(t))+2(\mathscr{D} x(t))^{T} D_{i} x\left(t-\tau_{3}(t)\right) \\
& \left.+x^{T}\left(t-\tau_{3}(t)\right) D_{i}^{T} D_{i} x\left(t-\tau_{3}(t)\right)\right\} \\
& =E(\mathscr{D} x(t))^{T}(\mathscr{D} x(t))+2 E(\mathscr{D} x(t))^{T} D_{i} x(t \\
& \left.-\tau_{3}(t)\right)+E x^{T}\left(t-\tau_{3}(t)\right) D_{i}^{T} D_{i} x\left(t-\tau_{3}(t)\right) .
\end{aligned}
$$

By utilizing Lemma 4 and (48), a positive scalar $\varepsilon$ can be found, such that

$$
\begin{aligned}
E x^{T} & (t) x(t) \leq(1+\varepsilon) E(\mathscr{D} x(t))^{T}(\mathscr{D} x(t)) \\
& +\left(1+\varepsilon^{-1}\right) \lambda_{\max }\left(D_{i}^{T} D_{i}\right) E x^{T}\left(t-\tau_{3}(t)\right) \\
& \cdot x\left(t-\tau_{3}(t)\right), \\
& \left(1+\varepsilon^{-1}\right) \lambda_{\max }\left(D_{i}^{T} D_{i}\right) e^{\gamma \tau_{3}}<1 .
\end{aligned}
$$

If $-1 \leq \alpha_{i}<0$, by using (44) and (49), for any $t^{*} \geq 0$, we can get the following result by the same derivation in [22]:

$$
\begin{aligned}
& \sup _{0 \leq t \leq t^{*}}\left\{E\left[x^{T}(t) x(t)\right] e^{\gamma t}\right\} \leq \frac{(1+\varepsilon) E V\left(0, r(0), x_{0}\right)}{\min _{i \in S} \lambda_{\min }\left(P_{i}\right)} \\
& +\left(1+\varepsilon^{-1}\right) \lambda_{\max }\left(D_{i}^{T} D_{i}\right) e^{\gamma \tau_{3}} \\
& \cdot \sup _{0 \leq t \leq t^{*}}\left\{E\left[x^{T}\left(t-\tau_{3}(t)\right) x\left(t-\tau_{3}(t)\right)\right] e^{\gamma\left(t-\tau_{3}(t)\right)}\right\} \\
& \leq \frac{(1+\varepsilon) E V\left(0, r(0), x_{0}\right)}{\min _{i \in S} \lambda_{\min }\left(P_{i}\right)}+\left(1+\varepsilon^{-1}\right) \\
& \cdot \lambda_{\max }\left(D_{i}^{T} D_{i}\right) e^{\gamma \tau_{3}}\left[\sup _{-\tau_{3}(t) \leq \theta<0} E\|\xi(\theta)\|^{2}\right. \\
& \left.+\sup _{0 \leq t \leq t^{*}}\left\{E\left[x^{T}(t) x(t)\right] e^{\gamma t}\right\}\right] \leq\left(1+\varepsilon^{-1}\right) \\
& \cdot \lambda_{\max }\left(D_{i}^{T} D_{i}\right) e^{\gamma \tau_{3}}\left[\sup _{-\tau \leq \theta \leq 0} E\|\xi(\theta)\|^{2}\right. \\
& \left.+\sup _{0 \leq t \leq t^{*}}\left\{E\left[x^{T}(t) x(t)\right] e^{\gamma t}\right\}\right] \\
& +\frac{(1+\varepsilon) E V\left(0, r(0), x_{0}\right)}{\min _{i \in S} \lambda_{\min }\left(P_{i}\right)}
\end{aligned}
$$

Because (46) and (50) hold, we have

$$
\sup _{0 \leq t \leq t^{*}}\left\{E\left[x^{T}(t) x(t)\right] e^{\gamma t}\right\} \leq \mathscr{M} \sup _{-\tau \leq \theta \leq 0} E\|\xi(\theta)\|^{2}
$$

where

$$
=\frac{\left((1+\varepsilon) M_{1} / \min _{i \in S} \lambda_{\min }\left(P_{i}\right)+\left(1+\varepsilon^{-1}\right) \lambda_{\max }\left(D_{i}^{T} D_{i}\right) e^{\gamma \tau_{3}}\right)}{\left(1-\left(1+\varepsilon^{-1}\right) \lambda_{\max }\left(D_{i}^{T} D_{i}\right) e^{\gamma \tau_{3}}\right)} .
$$

Letting $t^{*} \rightarrow \infty$ yields

$$
\sup _{t \in[0, \infty)}\left\{E\left[x^{T}(t) x(t)\right] e^{\gamma t}\right\} \leq \mathscr{M} \sup _{-\tau \leq \theta \leq 0} E\|\xi(\theta)\|^{2} .
$$

Obviously, for $-1 \leq \alpha_{i}<0, t \geq 0$,

$$
E x^{T}(t) x(t) \leq \mathscr{M} e^{-\gamma t} \sup _{-\tau \leq \theta \leq 0} E\|\xi(\theta)\|^{2} .
$$

Next, along the same line of (55), it can be deduced that for $\alpha_{i}>0, t \in\left[t_{k-1}, t_{k}\right), k \in \mathbb{N}^{+}$,

$$
\begin{aligned}
& E x^{T}(t) x(t) \\
& \leq \mathscr{M}^{\prime} e^{\left(-\gamma+\ln \alpha / \inf \left\{t_{k}-t_{k-1}\right\}\right) t} \sup _{-\tau \leq \theta \leq 0} E\|\xi(\theta)\|^{2},
\end{aligned}
$$

where 


$$
\mathscr{M}^{\prime}=\frac{\left((1+\varepsilon) M_{1} / \min _{i \in S} \lambda_{\min }\left(P_{i}\right)+\left(1+\varepsilon^{-1}\right) \lambda_{\max }\left(D_{i}^{T} D_{i}\right) e^{\left(\gamma-\ln \alpha / \inf \left\{t_{k}-t_{k-1}\right\}\right) \tau_{3}}\right)}{\left(1-\left(1+\varepsilon^{-1}\right) \lambda_{\max }\left(D_{i}^{T} D_{i}\right) e^{\left(\gamma-\ln \alpha / \inf \left\{t_{k}-t_{k-1}\right\}\right) \tau_{3}}\right)}
$$

Hence, for $\alpha_{i} \geq-1\left(\alpha_{i} \neq 0\right)$, by Definition 2 and (55) and (56), it can be seen that the trivial solution of system (10) is robustly exponentially stable in mean square. Moreover, the exponential convergence rate is

$$
\begin{gathered}
\gamma, \quad \text { if }-1 \leq \alpha_{i}<0, \\
\gamma-\frac{\ln \alpha}{\inf \left\{t_{k}-t_{k-1}\right\}}, \quad \text { if } \alpha_{i}>0 .
\end{gathered}
$$

This completes the proof of Theorem 7 .

Remark 8. In fact, exponential convergence rate of the trivial solution of system (10) is the inherent essence. The constructed exponential-type Lyapunov-Krasovskii functional in the proof of Theorem 7 is aimed at estimating a closely approximate exponential convergence rate of the trivial solution of system (10) mathematically.

Remark 9. When $-1 \leq \alpha_{i}<0$, the impulses are stabilizing; when $\alpha_{i}>0$, the impulses are destabilizing; and when $W_{i k}=I$, the impulses are neutral-type (i.e., they are neither helpful for stability of system (10) nor destabilizing). $\alpha_{i} \neq 0$ is necessary since the Markovian jumping would occur at the impulsive time instants; that is, $P_{i}$ is changing with the mode's change, and there always exist scalars $\alpha_{i}>0$ such that $P_{l} \leq\left(1+\alpha_{i}\right) P_{i}$. To the best of authors' knowledge, there is no result about dividing the impulses into three types for robust global exponential stability for impulsive stochastic neural networks of neutral-type with Markovian parameters, mixed time delays, and parametric uncertainties. Moreover, because the stability analysis for the case of neutral-type impulses is similar to that of destabilizing impulses, the robust exponential stability in mean square of system (10) has been classified into two categories: $-1 \leq \alpha_{i}<0$ and $\alpha_{i}>0$.

Remark 10. As shown in (58), the effects of the three types of impulses for the exponential convergence rate of the trivial solution of system (10) have been explicitly presented, which further verifies the characteristics of the different impulses.

When system (10) is without parametric uncertainties, by constructing the same Lyapunov-Krasovskii functional, from Theorem 7, the following corollary can be deduced to guarantee the exponential stability in mean square of the trivial solution of system (10).

Corollary 11. Assume that hypotheses (H1)-(H3) hold. For given scalars $h_{1}, h_{2}, \tau_{2}, \tau_{3}$, and $\mu_{1}, \mu_{2}, \mu_{3}$, the trivial solution of system (10) is exponentially stable in mean square if there exist positive scalars $\lambda_{i}, \alpha_{i} \geq-1\left(\alpha_{i} \neq 0\right), \alpha=\max \left\{1+\alpha_{i}\right\}(i \in S)$, $\gamma$, positive definite matrices $P_{i}(i \in S), Q_{1}, Q_{2}, Q_{3}, Q_{4}, Q_{5}, Q_{6}$, positive diagonal matrices $R_{i}, S_{i}(i \in S)$, and any real matrices $N_{q}(q=1,2, \ldots, 10)$ of appropriate dimensions such that

$$
\begin{aligned}
P_{i} & \leq \lambda_{i} I, \\
W_{i k}^{T} P_{l} W_{i k}-P_{i} & \leq \alpha_{i} P_{i} \quad\left[\text { here } r\left(t_{k}\right)=l\right], \\
\Phi_{i}^{\prime} & <0
\end{aligned}
$$

where

$$
\begin{aligned}
& \Phi_{i}^{\prime}=\left(\phi_{i m n}^{\prime}\right)_{13 \times 13}, \\
& m=1,2, \ldots, 13, n=1,2, \ldots, 13, \\
& \phi_{i 1,1}^{\prime}=-P_{i} C_{i}-C_{i}^{T} P_{i}+\gamma P_{i}+\lambda_{i} H_{1 i}+\sum_{j=1}^{N} \pi_{i j} P_{j} \\
& +e^{-\gamma h_{2}} Q_{1}+\sum_{j=2}^{3} e^{-\gamma \tau_{j}} Q_{j} \\
& +\frac{h_{2}-h_{1}}{\gamma}\left(e^{\gamma h_{2}}-e^{\gamma h_{1}}\right) Q_{5} \\
& +\frac{\tau_{3}}{\gamma}\left(e^{\gamma \tau_{3}}-1\right) Q_{6}-2 L_{1} R_{i} L_{2}+N_{1}+N_{1}^{T} \\
& +N_{6}+N_{6}^{T}, \\
& \phi_{i 1,2}^{\prime}=-N_{1}+N_{2}^{T} \text {, } \\
& \phi_{i 1,3}^{\prime}=-N_{6}+N_{7}^{T} \text {, } \\
& \phi_{i 1,4}^{\prime}=-\left(\sum_{j}^{N} \pi_{i j} P_{j}\right) D_{i}-\gamma P_{i} D_{i}+C_{i}^{T} P_{i} D_{i}-N_{1} D_{i} \\
& -N_{6} D_{i}+N_{3}^{T}+N_{8}^{T}, \\
& \phi_{i 1,5}^{\prime}=N_{1} D_{i}+N_{4}^{T} \text {, } \\
& \phi_{i 1,6}^{\prime}=N_{6} D_{i}+N_{9}^{T} \text {, } \\
& \phi_{i 1,7}^{\prime}=P_{i} A_{i}+\left(L_{1}+L_{2}\right) R_{i}, \\
& \phi_{i 1,8}^{\prime}=P_{i} B_{i} \text {, } \\
& \phi_{i 1,9}^{\prime}=P_{i} E_{i}, \\
& \phi_{i 1,10}^{\prime}=-N_{1}+N_{5}^{T} \text {, } \\
& \phi_{i 1,11}^{\prime}=-N_{6}+N_{10}^{T} \text {, }
\end{aligned}
$$




$$
\begin{aligned}
& \phi_{i 2,2}^{\prime}=\lambda_{i} H_{2 i}-\left(1-\mu_{1}\right) h\left(\mu_{1}\right) Q_{1}-2 L_{1} S_{i} L_{2}-N_{2} \\
& -N_{2}^{T} \\
& \phi_{i 2,4}^{\prime}=-N_{2} D_{i}-N_{3}^{T}, \\
& \phi_{i 2,5}^{\prime}=N_{2} D_{i}-N_{4}^{T} \text {, } \\
& \phi_{i 2,8}^{\prime}=\left(L_{1}+L_{2}\right) S_{i} \text {, } \\
& \phi_{i 2,10}^{\prime}=-N_{2}-N_{5}^{T} \text {, } \\
& \phi_{i 3,3}^{\prime}=\lambda_{i} H_{3 i}-\left(1-\mu_{2}\right) h\left(\mu_{2}\right) Q_{2}-N_{7}-N_{7}^{T}, \\
& \phi_{i 3,4}^{\prime}=-N_{7} D_{i}-N_{8}^{T}, \\
& \phi_{i 3,6}^{\prime}=N_{7} D_{i}-N_{9}^{T} \text {, } \\
& \phi_{i 3,11}^{\prime}=-N_{7}-N_{10}^{T} \text {, } \\
& \phi_{i 4,4}^{\prime}=\gamma D_{i}^{T} P_{i} D_{i}+D_{i}^{T}\left(\sum_{j=1}^{N} \pi_{i j} P_{j}\right) D_{i}+\lambda_{i} H_{4 i} \\
& -\left(1-\mu_{3}\right) h\left(\mu_{3}\right) Q_{3}-N_{3} D_{i}-D_{i}^{T} N_{3}^{T} \\
& -N_{8} D_{i}-D_{i}^{T} N_{8}^{T} \text {, } \\
& \phi_{i 4,5}^{\prime}=N_{3} D_{i}-D_{i}^{T} N_{4}^{T}, \\
& \phi_{i 4,6}^{\prime}=N_{8} D_{i}-D_{i}^{T} N_{9}^{T}, \\
& \phi_{i 4,7}^{\prime}=-D_{i}^{T} P_{i} A_{i}, \\
& \phi_{i 4,8}^{\prime}=-D_{i}^{T} P_{i} B_{i}, \\
& \phi_{i 4,9}^{\prime}=-D_{i}^{T} P_{i} E_{i}, \\
& \phi_{i 4,10}^{\prime}=-N_{3}-D_{i}^{T} N_{5}^{T} \text {, } \\
& \phi_{i 4,11}^{\prime}=-N_{8}-D_{i}^{T} N_{10}^{T}, \\
& \phi_{i 5,5}^{\prime}=N_{4} D_{i}+D_{i}^{T} N_{4}^{T}, \\
& \phi_{5,10}^{\prime}=-N_{4}+D_{i}^{T} N_{5}^{T}, \\
& \phi_{i 6,6}^{\prime}=N_{9} D_{i}+D_{i}^{T} N_{9}^{T}, \\
& \phi_{i 6,11}^{\prime}=-N_{9}+D_{i}^{T} N_{10}^{T}, \\
& \phi_{i 7,7}^{\prime}=\frac{\tau}{\gamma}\left(e^{\gamma \tau}-1\right) Q_{4}-2 R_{i}, \\
& \phi_{i 8,8}^{\prime}=-2 S_{i}, \\
& \phi_{i 9,9}^{\prime}=-Q_{4} \text {, } \\
& \phi_{i 10,10}^{\prime}=-N_{5}-N_{5}^{T}, \\
& \phi_{i 11,11}^{\prime}=-N_{10}-N_{10}^{T} \text {, }
\end{aligned}
$$$$
\begin{aligned}
& \phi_{i 12,12}^{\prime}=-Q_{5}, \\
& \phi_{i 13,13}^{\prime}=-Q_{6},
\end{aligned}
$$

and the function $h(u) \in \mathbb{R}^{+}, u \in \mathbb{R}$, is defined as

$$
h(u)= \begin{cases}1, & u>1 \\ e^{-2 \gamma \tau}, & u \leq 1\end{cases}
$$

And for $\alpha_{i}>0,-\gamma+\ln \alpha / \inf \left\{t_{k}-t_{k-1}\right\}<0, k \in \mathbb{N}^{+}$, other elements of $\Phi_{i}^{\prime}$ are all equal to 0.

When system (10) is without Markovian jumping parameters, parametric uncertainties, distributed time-varying delay, impulses, and stochastic perturbation, then system (10) can be written as

$$
\begin{aligned}
d & {\left[x(t)-D x\left(t-\tau_{3}(t)\right)\right] } \\
& =\left[-C x(t)+A g(x(t))+B g\left(x\left(t-\tau_{1}(t)\right)\right)\right] d t .
\end{aligned}
$$

Construct a Lyapunov-Krasovskii functional as follows:

$$
\begin{aligned}
& V(t, x(t))=e^{\gamma t}\left(x(t)-D x\left(t-\tau_{3}(t)\right)\right)^{T} \\
& \cdot P\left(x(t)-D x\left(t-\tau_{3}(t)\right)\right) \\
& +\int_{t-\tau_{1}(t)}^{t} e^{\gamma\left(s-h_{2}\right)} x^{T}(s) Q_{1} x(s) d s \\
& +\int_{t-\tau_{3}(t)}^{t} e^{\gamma\left(s-\tau_{3}\right)} x^{T}(s) Q_{2} x(s) d s+\left(h_{2}-h_{1}\right) \\
& \cdot \int_{-h_{2}}^{-h_{1}} \int_{t+\beta}^{t} e^{\gamma(s-\beta)} x^{T}(s) Q_{3} x(s) d s d \beta \\
& +\tau_{3} \int_{-\tau_{3}}^{0} \int_{t+\beta}^{t} e^{\gamma(s-\beta)} x^{T}(s) Q_{4} x(s) d s d \beta .
\end{aligned}
$$

From Theorem 7, the following corollary can be deduced to guarantee the exponential stability of the trivial solution of system (62).

Corollary 12. Assume that hypotheses (H1)-(H2) hold. For given scalars $h_{1}, h_{2}, \tau_{3}$, and $\mu_{1}, \mu_{3}$, the trivial solution of system (62) is exponentially stable if there exist positive scalar $\gamma$, positive definite matrices $P, Q_{1}, Q_{2}, Q_{3}, Q_{4}$, positive diagonal matrices $R, S$, and any real matrices $N_{q}(q=1,2, \ldots, 5)$ of appropriate dimensions such that

$$
\Phi^{\prime}<0,
$$


where

$$
\begin{aligned}
\Phi^{\prime}= & \left(\phi_{i m n}^{\prime}\right)_{9 \times 9}, \quad m=1,2, \ldots, 9, n=1,2, \ldots, 9, \\
\phi_{1,1}^{\prime}= & -P C-C^{T} P+\gamma P+e^{-\gamma h_{2}} Q_{1}+e^{-\gamma \tau_{3}} Q_{2} \\
& +\frac{h_{2}-h_{1}}{\gamma}\left(e^{\gamma h_{2}}-e^{\gamma h_{1}}\right) Q_{3} \\
& +\frac{\tau_{3}}{\gamma}\left(e^{\gamma \tau_{3}}-1\right) Q_{4}-2 L_{1} R L_{2}+N_{1}+N_{1}^{T}, \\
\phi_{1,2}^{\prime}= & -N_{1}+N_{2}^{T}, \\
\phi_{1,3}^{\prime}= & -\gamma P D+C^{T} P D-N_{1} D+N_{3}^{T}, \\
\phi_{1,4}^{\prime}= & N_{1} D+N_{4}^{T}, \\
\phi_{1,5}^{\prime}= & P A+\left(L_{1}+L_{2}\right) R, \\
\phi_{1,6}^{\prime}= & P B \\
\phi_{1,7}^{\prime}= & -N_{1}+N_{5}^{T}, \\
\phi_{2,2}^{\prime}= & -\left(1-\mu_{1}\right) h\left(\mu_{1}\right) Q_{1}-2 L_{1} S L_{2}-N_{2}-N_{2}^{T}, \\
\phi_{2,3}^{\prime}= & -N_{2} D-N_{3}^{T}, \\
\phi_{2,4}^{\prime}= & N_{2} D-N_{4}^{T}, \\
\phi_{2,6}^{\prime}= & \left(L_{1}+L_{2}\right) S, \\
\phi_{2,7}^{\prime}= & -N_{2}-N_{5}^{T}, \\
\phi_{3,3}^{\prime}= & \gamma D^{T} P D-\left(1-\mu_{3}\right) h\left(\mu_{3}\right) Q_{2}-N_{3} D-D^{T} N_{3}^{T}, \\
\phi_{3,4}^{\prime}= & N_{3} D-D^{T} N_{4}^{T}, \\
\phi_{3,5}^{\prime}= & -D^{T} P A, \\
\phi_{3,6}^{\prime}= & -D^{T} P B, \\
\phi_{3,7}^{\prime}= & -N_{3}-D^{T} N_{5}^{T}, \\
\phi_{4,4}^{\prime}= & N_{4} D+D^{T} N_{4}^{T}, \\
\phi_{4,7}^{\prime}= & -N_{4}+D^{T} N_{5}^{T}, \\
\phi_{5,5}^{\prime}= & -2 R \\
\phi_{6,6}^{\prime}= & -2 S, \\
\phi_{7,7}^{\prime}= & -N_{5}-Q_{5}^{T}, \\
\hline & -Q_{4}, \\
&
\end{aligned}
$$

and the function $h(u) \in \mathbb{R}^{+}, u \in \mathbb{R}$, is defined as

$$
h(u)= \begin{cases}1, & u>1, \\ e^{-2 \gamma \tau}, & u \leq 1 .\end{cases}
$$

Consider 2D delayed impulsive stochastic neural networks of neutral-type (10) with Markovian switching and parametric uncertainties:

$$
\begin{aligned}
& C_{1}=\left(\begin{array}{cc}
2.9 & 0 \\
0 & 2.8
\end{array}\right), \\
& C_{2}=\left(\begin{array}{cc}
2.5 & 0 \\
0 & 2.6
\end{array}\right), \\
& A_{1}=\left(\begin{array}{ll}
0.2 & 0.18 \\
0.3 & 0.19
\end{array}\right), \\
& A_{2}=\left(\begin{array}{ll}
0.3 & 0 \\
0.4 & 0
\end{array}\right), \\
& B_{1}=\left(\begin{array}{ll}
0.8 & 0.2 \\
0.2 & 0.3
\end{array}\right), \\
& B_{2}=\left(\begin{array}{cc}
2.5 & 1.5 \\
1 & 2.5
\end{array}\right) \text {, } \\
& D_{1}=\left(\begin{array}{cc}
0.2 & 0 \\
0 & 0.2
\end{array}\right) \text {, } \\
& D_{2}=\left(\begin{array}{cc}
0.3 & 0 \\
0 & 0.3
\end{array}\right) \text {, } \\
& E_{1}=\left(\begin{array}{cc}
4 & 0.04 \\
0.14 & 4
\end{array}\right), \\
& E_{2}=\left(\begin{array}{cc}
4 & 1.5 \\
1 & 4
\end{array}\right), \\
& Z_{1}=\left(\begin{array}{cc}
0.1 & -0.2 \\
0.7 & 0.2
\end{array}\right), \\
& Z_{2}=\left(\begin{array}{cc}
-0.1 & -0.2 \\
-0.1 & 0.2
\end{array}\right), \\
& H_{1}=\left(\begin{array}{ll}
-0.3 & 0.1 \\
-0.2 & 0.1
\end{array}\right) \text {, } \\
& H_{2}=\left(\begin{array}{ll}
0.3 & -0.4 \\
0.7 & -0.1
\end{array}\right) \text {, }
\end{aligned}
$$

\section{Numerical Results}

In this section, two numerical examples are presented to

Example 13 (see [13]). Let the state space of Markov chain

$$
\Pi=\left[\begin{array}{cc}
-0.45 & 0.45 \\
0.5 & -0.5
\end{array}\right]
$$
. 


$$
\begin{aligned}
& J_{1}=\left(\begin{array}{cc}
-0.5 & -0.4 \\
0.2 & -0.2
\end{array}\right) \\
& J_{2}=\left(\begin{array}{cc}
-0.1 & -0.4 \\
0.4 & 0.3
\end{array}\right), \\
& K_{1}=\left(\begin{array}{cc}
-0.2 & 0.2 \\
0.1 & 0.8
\end{array}\right) \text {, } \\
& K_{2}=\left(\begin{array}{cc}
0.1 & 0.3 \\
-0.4 & -0.3
\end{array}\right) \text {, } \\
& F_{1}(t)=\left(\begin{array}{cc}
\sin (t) & 0 \\
0 & \cos (t)
\end{array}\right), \\
& F_{2}(t)=\left(\begin{array}{cc}
\cos (t) & 0 \\
0 & \sin (t)
\end{array}\right), \\
& g(x(t))=\tanh (x(t)), \\
& \tau_{1}(t)=0.6+0.6 \sin (2 t), \\
& \tau_{2}(t)=0.25+0.25 \cos (4 t), \\
& \tau_{3}(t)=1.5+1.5 \cos (t), \\
& \sigma_{1}(t)=\sigma_{2}(t) \\
& =\left(\begin{array}{cc}
0.3 x_{1}(t) & 0 \\
0 & 0.2 x_{2}\left(t-\tau_{1}(t)\right)
\end{array}\right) \\
& +\left(\begin{array}{cc}
0.3 x_{2}(t) & 0 \\
0 & 0.2 x_{2}\left(t-\tau_{2}(t)\right)
\end{array}\right) \\
& +\left(\begin{array}{cc}
0.3 x_{1}\left(t-\tau_{2}(t)\right) & 0 \\
0 & 0.2 x_{2}\left(t-\tau_{3}(t)\right)
\end{array}\right) .
\end{aligned}
$$

Then system (10) satisfies hypotheses $(H 1)-(H 3)$ with

$$
\begin{aligned}
h_{1} & =0, \\
h_{2} & =1.2, \\
\tau_{2} & =0.5, \\
\tau_{3} & =3, \\
\mu_{1} & =1.2, \\
\mu_{2} & =1, \\
\mu_{3} & =1.5, \\
\tau & =4.2, \\
H_{11} & =H_{12}=0.18 I, \\
H_{21} & =H_{22}=0.08 I,
\end{aligned}
$$

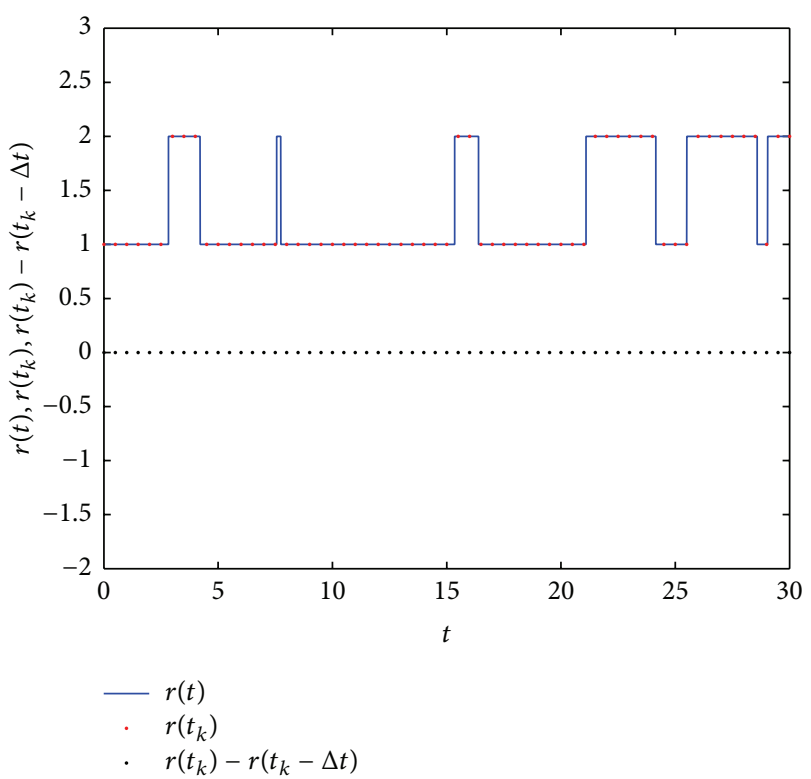

FIGURE 1: The 2-state Markov chain with $t_{k}=0.5+t_{k-1}, k \in \mathbb{N}^{+}$, $\Delta t=0.001$ in Example 13 .

$$
\begin{aligned}
H_{31} & =H_{32}=0.18 I, \\
H_{41} & =H_{42}=0.08 I, \\
L_{1} & =0, \\
L_{2} & =I, \\
L & =I .
\end{aligned}
$$

Case of the Stabilizing Impulses. Study the following impulsive gain matrices:

$$
\begin{aligned}
& W_{1 k}=\left(\begin{array}{cc}
0.9 & 0 \\
0 & 0.9
\end{array}\right), \\
& W_{2 k}=\left(\begin{array}{cc}
0.9 & 0 \\
0 & 0.9
\end{array}\right), \\
& \quad k \in \mathbb{N}^{+} .
\end{aligned}
$$

By choosing $\alpha_{1}=-0.1, \alpha_{2}=-0.1$, then the impulses are the stabilizing impulses. We set $t_{k}=0.5+t_{k-1}, k \in \mathbb{N}^{+}, \Delta t=0.001$. The 2-state Markov chain with $r(0)=1$ is shown in Figure 1, among which the right continuous Markov chain $\{r(t), t \geq 0\}$ is denoted by the solid blue line, and the Markov chain of the impulsive time instants $\left\{r\left(t_{k}\right), k \in \mathbb{N}^{+}\right\}$is denoted by the red point, and the black point is used to judge whether the Markovian jumping occurs at the impulsive time instants, that is, $r\left(t_{k}\right)-r\left(t_{k}-\Delta t\right)$. From Figure 1 , we can conclude that the Markovian jumping does not occur at the impulsive time instants when $t_{k}=0.5+t_{k-1}, k \in \mathbb{N}^{+}, \Delta t=0.001$.

By using the LMI toolbox in MATLAB, we search the maximum exponential convergence rate which is 5.4297 
subject to the LMIs (18)-(20). Let $\gamma=0.5$; we can obtain the following feasible solutions to the LMIs (18)-(20) in Theorem 7:

$$
\begin{aligned}
& P_{1}=\left(\begin{array}{cc}
0.0019 & -0.0001 \\
-0.0001 & 0.0010
\end{array}\right) \text {, } \\
& P_{2}=\left(\begin{array}{cc}
0.0018 & -0.0010 \\
-0.0010 & 0.0023
\end{array}\right) \text {, } \\
& Q_{1}=\left(\begin{array}{cc}
0.0229 & -0.0002 \\
-0.0002 & 0.0214
\end{array}\right) \text {, } \\
& Q_{2}=\left(\begin{array}{cc}
0.0192 & -0.0002 \\
-0.0002 & 0.0177
\end{array}\right) \text {, } \\
& Q_{3}=\left(\begin{array}{cc}
0.0089 & 0 \\
0 & 0.0091
\end{array}\right) \text {, } \\
& Q_{4}=\left(\begin{array}{ll}
0.0020 & 0.0001 \\
0.0001 & 0.0027
\end{array}\right), \\
& Q_{5}=\left(\begin{array}{cc}
0.0231 & -0.0003 \\
-0.0003 & 0.0213
\end{array}\right) \text {, } \\
& Q_{6}=\left(\begin{array}{cc}
0.0023 & 0 \\
0 & 0.0021
\end{array}\right) \text {, } \\
& R_{1}=\left(\begin{array}{cc}
0.1752 & 0 \\
0 & 0.1752
\end{array}\right) \text {, } \\
& R_{2}=\left(\begin{array}{cc}
0.1703 & 0 \\
0 & 0.1703
\end{array}\right) \text {, } \\
& S_{1}=\left(\begin{array}{cc}
0.1399 & 0 \\
0 & 0.1399
\end{array}\right) \text {, } \\
& S_{2}=\left(\begin{array}{cc}
0.1356 & 0 \\
0 & 0.1356
\end{array}\right) \text {, } \\
& N_{1}=\left(\begin{array}{rr}
-0.2415 & -0.0016 \\
-0.0016 & -0.2542
\end{array}\right) \text {, } \\
& N_{2}=\left(\begin{array}{ll}
0.2773 & 0.0014 \\
0.0016 & 0.2885
\end{array}\right), \\
& N_{3}=\left(\begin{array}{ll}
0.0934 & 0.0005 \\
0.0005 & 0.0965
\end{array}\right) \text {, } \\
& N_{4}=\left(\begin{array}{rr}
-0.0946 & -0.0002 \\
-0.0002 & -0.0968
\end{array}\right) \text {, } \\
& N_{5}=\left(\begin{array}{ll}
0.2595 & 0.0006 \\
0.0005 & 0.2638
\end{array}\right) \text {, }
\end{aligned}
$$

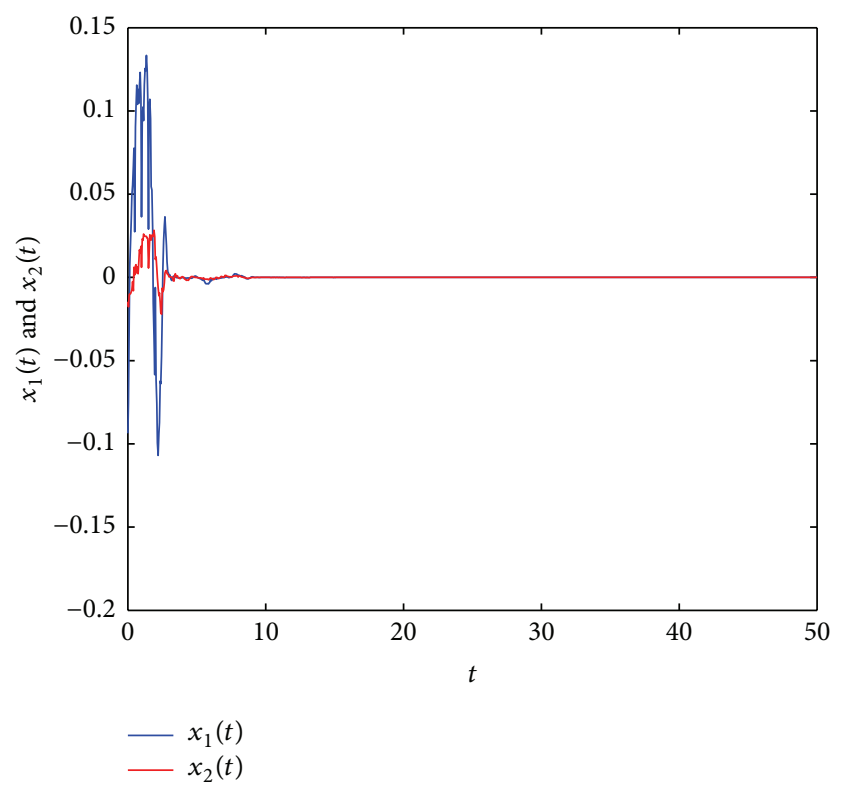

FIGURE 2: The dynamic behavior of system (10) with the stabilizing impulses, with the initial condition of every state uniformly randomly selected from $[-0.1 ; 0.1], s \in[-4.2,0]$ in Example 13 .

$$
\begin{aligned}
N_{6} & =\left(\begin{array}{rr}
-0.2368 & -0.0016 \\
-0.0016 & -0.2497
\end{array}\right), \\
N_{7} & =\left(\begin{array}{ll}
0.2395 & 0.0015 \\
0.0015 & 0.2517
\end{array}\right) \\
N_{8} & =\left(\begin{array}{ll}
0.0923 & 0.0007 \\
0.0007 & 0.0964
\end{array}\right) \\
N_{9} & =\left(\begin{array}{ll}
-0.0947 & -0.0003 \\
-0.0003 & -0.0974
\end{array}\right) \\
N_{10} & =\left(\begin{array}{ll}
0.2581 & 0.0007 \\
0.0007 & 0.2635
\end{array}\right) \\
\lambda_{1} & =0.0677 \\
\lambda_{2} & =0.0816, \\
\kappa_{1} & =0.0015 \\
\kappa_{2} & =0.0015
\end{aligned}
$$

Set the simulation step size $h=0.05$ and $r(0)=1$, $\Delta t=0.001$. The dynamic behavior of system (10) with the stabilizing impulses in Example 13 is presented in Figure 2, with the initial condition of every state uniformly randomly selected from $[-0.1 ; 0.1], s \in[-4.2,0]$. Therefore, it can be verified that system (10) with the stabilizing impulses is robustly exponentially stable in mean square with exponential convergence rate 0.5 . 


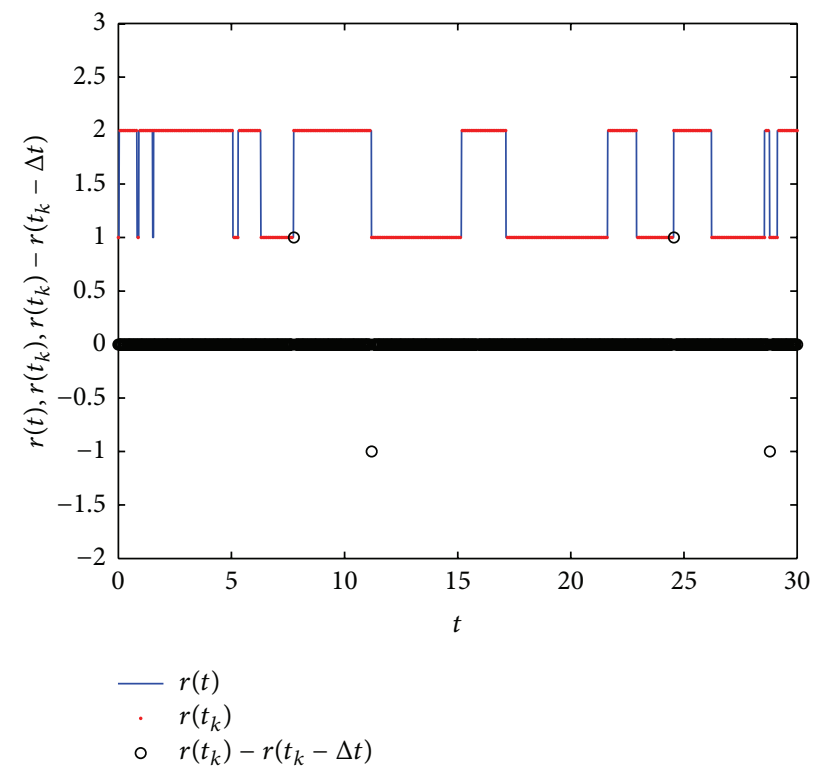

Figure 3: The 2-state Markov chain with $t_{k}=0.08+t_{k-1}, k \in \mathbb{N}^{+}$, $\Delta t=0.01$ in Example 13 .

Case of the Destabilizing Impulses. Study the following impulsive gain matrices:

$$
\begin{aligned}
& W_{1 k}=\left(\begin{array}{cc}
1.08 & 0 \\
0 & 1.08
\end{array}\right), \\
& W_{2 k}=\left(\begin{array}{cc}
1.08 & 0 \\
0 & 1.08
\end{array}\right), \\
& k \in \mathbb{N}^{+} .
\end{aligned}
$$

By choosing $\alpha_{1}=0.5, \alpha_{2}=0.5$, then the impulses are the destabilizing impulses. In order to find the maximum exponential convergence rate, we first assume that the Markovian jumping may occur at the impulsive time instants. By using the LMI toolbox in MATLAB, we search the maximum exponential convergence rate which is 5.4020 subject to the LMIs (18)-(20), and $\inf \left\{t_{k}-t_{k-1}\right\}>\ln (1.5) / 5.4020=0.0751$. Then set $t_{k}=0.08+t_{k-1}, k \in \mathbb{N}^{+}, \Delta t=0.01$. The 2-state Markov chain with $r(0)=1$ is shown in Figure 3, among which the right continuous Markov chain $\{r(t), t \geq 0\}$ is denoted by the solid blue line, and the Markov chain of the impulsive time instants $\left\{r\left(t_{k}\right), k \in \mathbb{N}^{+}\right\}$is denoted by the red point, and the black circle is used to judge whether the Markovian jumping occurs at the impulsive time instants, that is, $r\left(t_{k}\right)-r\left(t_{k}-\Delta t\right)$. From Figure 3 , we can conclude that the Markovian jumping would occur at the impulsive time instants when $t_{k}=0.08+t_{k-1}, k \in \mathbb{N}^{+}, \Delta t=0.01$, which further verify the correctness of the assumption.

Set the simulation step size $h=0.04$ and $r(0)=1$, $\Delta t=0.01$. The dynamic behavior of system (10) with the destabilizing impulses in Example 13 is presented in Figure 4, with the initial condition of every state uniformly randomly selected from $[-0.1 ; 0.1], s \in[-4.2,0]$. Therefore, it can be verified that system (10) with the destabilizing impulses is robustly exponentially stable in mean square.

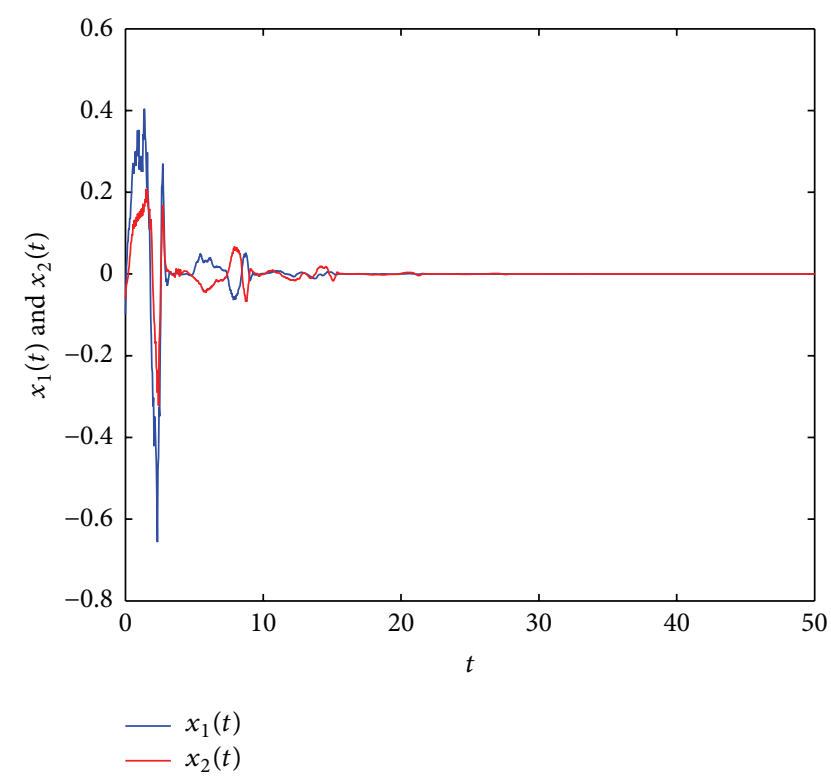

FIGURE 4: The dynamic behavior of system (10) with the destabilizing impulses, with the initial condition of every state uniformly randomly selected from $[-0.1 ; 0.1], s \in[-4.2,0]$ in Example 13 .

Case of the Neural-Type Impulses. Study the following impulsive gain matrices:

$$
\begin{aligned}
& W_{1 k}=\left(\begin{array}{ll}
1 & 0 \\
0 & 1
\end{array}\right), \\
& W_{2 k}=\left(\begin{array}{ll}
1 & 0 \\
0 & 1
\end{array}\right),
\end{aligned}
$$

$$
k \in \mathbb{N}^{+} .
$$

By choosing $\alpha_{1}=1, \alpha_{2}=1$, then the impulses are the neutraltype impulses. In order to find the maximum exponential convergence rate, we first assume that the Markovian jumping may occur at the impulsive time instants. By using the LMI toolbox in MATLAB, we search the maximum exponential convergence rate which is 5.4039 subject to the LMIs (19)(20), and $\inf \left\{t_{k}-t_{k-1}\right\}>\ln (2) / 5.4039=0.1283$. Then we set $t_{k}=0.15+t_{k-1}, k \in \mathbb{N}^{+}, \Delta t=0.01$. The 2-state Markov chain with $r(0)=1$ is shown in Figure 5, among which the right continuous Markov chain $\{r(t), t \geq 0\}$ is denoted by the solid blue line, and the Markov chain of the impulsive time instants $\left\{r\left(t_{k}\right), k \in \mathbb{N}^{+}\right\}$is denoted by the red point, and the black circle is used to judge whether the Markovian jumping occurs at the impulsive time instants, that is, $r\left(t_{k}\right)-r\left(t_{k}-\Delta t\right)$. From Figure 5, we can conclude that the Markovian jumping would occur at the impulsive time instants when $t_{k}=0.15+t_{k-1}$, $k \in \mathbb{N}^{+}, \Delta t=0.01$, which further verify the correctness of the assumption.

Set the simulation step size $h=0.05$ and $r(0)=1$, $\Delta t=0.01$. The dynamic behavior of system (10) with the neutral-type impulses in Example 13 is presented in Figure 6, with the initial condition of every state uniformly randomly selected from $[-0.1 ; 0.1], s \in[-4.2,0]$. Therefore, it can be 


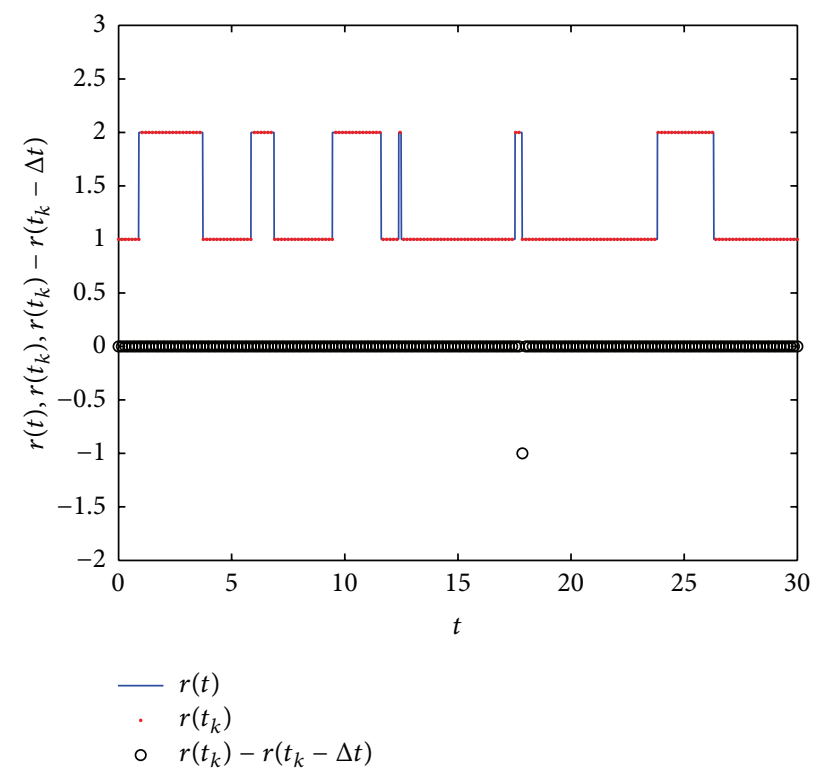

Figure 5: The 2-state Markov chain with $t_{k}=0.15+t_{k-1}, k \in \mathbb{N}^{+}$, $\Delta t=0.01$ in Example 13 .

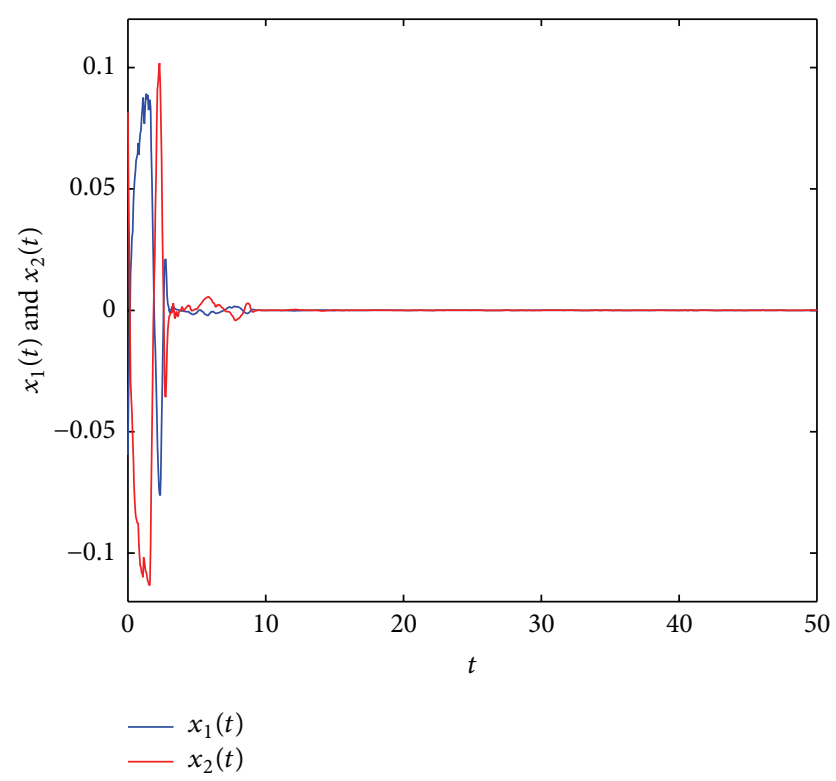

Figure 6: The dynamic behavior of system (10) with the neutraltype impulses, with the initial condition of every state uniformly randomly selected from $[-0.1 ; 0.1], s \in[-4.2,0]$ in Example 13.

verified that system (10) with the neutral-type impulses is robustly exponentially stable in mean square.

Example 14 (see [16]). Consider 2D delayed neural networks of neutral-type (62):

$$
\begin{aligned}
& C=\left(\begin{array}{ll}
5 & 0 \\
0 & 5
\end{array}\right), \\
& A=\left(\begin{array}{cc}
1 & 1 \\
1 & -1
\end{array}\right),
\end{aligned}
$$

TABLE 1: The maximum allowable delay bound (MADB) $h_{2}$ for different values of $\gamma$.

\begin{tabular}{lccc}
\hline Methods & $\gamma$ & 0.2000 & 1.3800 \\
\hline Example 1 in [16] & $h_{2}$ & 22.2000 & 1.0000 \\
Corollary 12 & $h_{2}$ & 88.1009 & 15.6047 \\
\hline
\end{tabular}

$$
\begin{aligned}
B & =\left(\begin{array}{cc}
1 & 1 \\
1 & -1
\end{array}\right), \\
D & =\left(\begin{array}{cc}
-0.5 & 0 \\
0 & -0.5
\end{array}\right), \\
g(x(t)) & =\left(0.25 \tanh \left(x_{1}(t)\right), 0.25 \tanh \left(x_{2}(t)\right)\right)^{T}, \\
\tau_{1}(t) & =0.5 \tau^{\prime}+0.5 \tau^{\prime} \cos \left(\frac{1}{\tau^{\prime}} t\right), \quad \tau^{\prime}>0, \\
\tau_{3}(t) & =1 .
\end{aligned}
$$

Then system (64) satisfies hypotheses $(H 1)-(H 2)$ with

$$
\begin{aligned}
h_{1} & =0, \\
h_{2} & =\tau^{\prime}, \\
\tau_{3} & =1, \\
\mu_{1} & =0.5, \\
\mu_{3} & =0, \\
\tau & =\tau^{\prime}+1, \\
L_{1} & =0, \\
L_{2} & =\operatorname{diag}(0.25,0.25), \\
L & =\operatorname{diag}(0.25,0.25) .
\end{aligned}
$$

By using the LMI toolbox in MATLAB, we search for the fact that the LMI (64) in Corollary 12 is feasible for any $\gamma \leq$ 12.5883 and $\tau^{\prime} \leq 2.0000$. A comparison of the maximum upper delay bound (MADB) $h_{2}$ for different values of $\gamma$ that guarantee the exponential stability of system (62) is made in Table 1 from which we can see that for this system of Example 14, the results in this paper are less conservative than that in [16].

\section{Conclusion}

In this paper, delay-dependent robust exponential stability criteria for a class of uncertain impulsive stochastic neural networks of neutral-type with Markovian parameters and mixed time-varying delays have been derived by the use of the Lyapunov-Krasovskii functional method, Jensen integral inequality, free-weight matrix method, and the LMI framework. The proposed results do not require the derivatives of discrete and distributed time-varying delays to be 0 or smaller than 1. Moreover, the main contribution of the 
proposed approach compared with related methods lies in the use of three types of impulses. Finally, two numerical examples are worked out to demonstrate the effectiveness and less conservativeness of our theoretical results over existing literature. One of our future research directions is to apply the proposed method to study the synchronization problem for Markovian jumping chaotic delayed neural networks of neutral-type via impulsive control.

\section{Competing Interests}

The authors declare that there is no conflict of interests regarding the publication of this paper.

\section{Acknowledgments}

This work was supported by the Opening Project of Sichuan Province University Key Laboratory of Bridge NonDestruction Detecting and Engineering Computing under Grant no. 2014QZJ01 and Grant no. 2015QYJ01 and National Natural Science Foundation of China under Grant 61573010 and Grant 11501391.

\section{References}

[1] H. Zhang, Z. Wang, and D. Liu, "A comprehensive review of stability analysis of continuous-time recurrent neural networks," IEEE Transactions on Neural Networks and Learning Systems, vol. 25, no. 7, pp. 1229-1262, 2014.

[2] J. Cao, R. Rakkiyappan, K. Maheswari et al., "Exponential $H_{\infty}$ filtering analysis for discrete-time switched neural networks with random delays using sojourn probabilities," Science China Technological Sciences, vol. 59, no. 3, pp. 387-402, 2016.

[3] R. Rakkiyappan, S. Dharani, and J. Cao, "Synchronization of neural networks with control packet loss and time-varying delay via stochastic sampled-data controller," IEEE Transactions on Neural Networks and Learning Systems, vol. 26, no. 12, pp. 3215-3226, 2015.

[4] J. Cao, R. Sivasamy, and R. Rakkiyappan, "Sampled-data $H_{\infty}$ synchronization of chaotic Lur/e systems with time delay," Circuits Systems \& Signal Process, vol. 35, pp. 811-835, 2016.

[5] Q. Zhu, J. Cao, and R. Rakkiyappan, "Exponential input-to-state stability of stochastic Cohen-Grossberg neural networks with mixed delays," Nonlinear Dynamics, vol. 79, no. 2, pp. 1085-1098, 2015.

[6] Q. Zhu, R. Rakkiyappan, and A. Chandrasekar, "Stochastic stability of Markovian jump BAM neural networks with leakage delays and impulse control," Neurocomputing, vol. 136, pp. 136151, 2014.

[7] Q. Zhu and J. Cao, "Exponential stability of stochastic neural networks with both Markovian jump parameters and mixed time delays," IEEE Transactions on Systems, Man, and Cybernetics Part B: Cybernetics, vol. 41, no. 2, pp. 341-353, 2011.

[8] Q. Zhu and J. Cao, "Stability analysis for stochastic neural networks of neutral type with both Markovian jump parameters and mixed time delays," Neurocomputing, vol. 73, no. 13-15, pp. 2671-2680, 2010.

[9] O. M. Kwon, J. H. Park, S. M. Lee, and E. J. Cha, "Analysis on delay-dependent stability for neural networks with time-varying delays," Neurocomputing, vol. 103, no. 2, pp. 114120, 2013.

[10] G. Zhang, X. Lin, and X. Zhang, "Exponential stabilization of neutral-type neural networks with mixed interval time-varying delays by intermittent control: a CCL approach," Circuits, Systems, and Signal Processing, vol. 33, no. 2, pp. 371-391, 2014.

[11] H. Bao and J. Cao, "Stochastic global exponential stability for neutral-type impulsive neural networks with mixed timedelays and Markovian jumping parameters," Communications in Nonlinear Science and Numerical Simulation, vol. 16, no. 9, pp. 3786-3791, 2011.

[12] H. Zhang, M. Dong, Y. Wang, and N. Sun, "Stochastic stability analysis of neutral-type impulsive neural networks with mixed time-varying delays and Markovian jumping," Neurocomputing, vol. 73, no. 13-15, pp. 2689-2695, 2010.

[13] Y. Gao, W. Zhou, C. Ji, D. Tong, and J. Fang, "Globally exponential stability of stochastic neutral-type delayed neural networks with impulsive perturbations and Markovian switching," Nonlinear Dynamics, vol. 70, no. 3, pp. 2107-2116, 2012.

[14] C.-D. Zheng, Y. Gu, W. Liang, and Z. Wang, "Novel delaydependent stability criteria for switched Hopfield neural networks of neutral type," Neurocomputing, vol. 158, pp. 117-126, 2015.

[15] J. Xia, J. H. Park, and H. Zeng, "Improved delay-dependent robust stability analysis for neutral-type uncertain neural networks with Markovian jumping parameters and time-varying delays," Neurocomputing, vol. 149, pp. 1198-1205, 2015.

[16] C.-H. Lien, K.-W. Yu, Y.-F. Lin, Y.-J. Chung, and L.-Y. Chung, "Global exponential stability for uncertain delayed neural networks of neutral type with mixed time delays," IEEE Transactions on Systems, Man, and Cybernetics, Part B: Cybernetics, vol. 38, no. 3, pp. 709-720, 2008.

[17] H. Zhang, Z. Liu, and G.-B. Huang, "Novel delay-dependent robust stability analysis for switched neutral-type neural networks with time-varying delays via SC technique," IEEE Transactions on Systems, Man, and Cybernetics, Part B: Cybernetics, vol. 40, no. 6, pp. 1480-1491, 2010.

[18] S. Lakshmanan, J. H. Park, H. Y. Jung, O. M. Kwon, and R. Rakkiyappan, "A delay partitioning approach to delaydependent stability analysis for neutral type neural networks with discrete and distributed delays," Neurocomputing, vol. 111, no. 6, pp. 81-89, 2013.

[19] W. Zhou, Q. Zhu, P. Shi, H. Su, J. Fang, and L. Zhou, "Adaptive synchronization for neutral-type neural networks with stochastic perturbation and Markovian switching parameters," IEEE Transactions on Cybernetics, vol. 44, no. 12, pp. 2848-2860, 2014.

[20] Q. Zhu, W. Zhou, L. Zhou, M. Wu, and D. Tong, "Modedependent projective synchronization for neutral-type neural networks with distributed time-delays," Neurocomputing, vol. 140, pp. 97-103, 2014.

[21] X. Li, "Global robust stability for stochastic interval neural networks with continuously distributed delays of neutral type," Applied Mathematics and Computation, vol. 215, no. 12, pp. 4370-4384, 2010.

[22] Y. Zhang, D.-W. Gu, and S. Xu, "Global exponential adaptive synchronization of complex dynamical networks with neutraltype neural network nodes and stochastic disturbances," IEEE Transactions on Circuits and Systems I: Regular Papers, vol. 60, no. 10, pp. 2709-2718, 2013.

[23] F. Deng, Q. Luo, and X. Mao, "Stochastic stabilization of hybrid differential equations," Automatica, vol. 48, no. 9, pp. 2321-2328, 2012. 
[24] X. Mao, G. G. Yin, and C. Yuan, "Stabilization and destabilization of hybrid systems of stochastic differential equations," Automatica, vol. 43, no. 2, pp. 264-273, 2007.

[25] S. Blythe, X. Mao, and X. Liao, "Stability of stochastic delay neural networks," Journal of the Franklin Institute, vol. 338, no. 4, pp. 481-495, 2001.

[26] Q. Zhu and J. Cao, "Mean-square exponential input-to-state stability of stochastic delayed neural networks," Neurocomputing, vol. 131, pp. 157-163, 2014.

[27] V. Lakshmikantham, D. D. Bainov, and P. S. Simeonov, Theory of Impulsive Differential Equations, World Scientific, Singapore, 1989.

[28] H. Zhang, T. Ma, G.-B. Huang, and Z. Wang, "Robust global exponential synchronization of uncertain chaotic delayed neural networks via dual-stage impulsive control," IEEE Transactions on Systems, Man, and Cybernetics, Part B: Cybernetics, vol. 40, no. 3, pp. 831-844, 2010.

[29] H. Li, B. Chen, Q. Zhou, and S. Fang, "Robust exponential stability for uncertain stochastic neural networks with discrete and distributed time-varying delays," Physics Letters A, vol. 372, no. 19, pp. 3385-3394, 2008.

[30] T. Huang, C. Li, S. Duan, and J. A. Starzyk, "Robust exponential stability of uncertain delayed neural networks with stochastic perturbation and impulse effects," IEEE Transactions on Neural Networks and Learning Systems, vol. 23, no. 6, pp. 866-875, 2012.

[31] N. N. Krasovskii and A. Lidskii, "Analytical design of controllers in systems with random attributes. I. Statement of the problem, method of solving," Automation and Remote Control, vol. 22, pp. 1021-1025, 1961.

[32] C.-D. Zheng, Y. Wang, and Z. Wang, "New stability results of neutral-type neural networks with continuously distributed delays and impulses," International Journal of Computer Mathematics, vol. 91, no. 9, pp. 1880-1896, 2014.

[33] Q. Zhu and J. Cao, "Stability of Markovian jump neural networks with impulse control and time varying delays," Nonlinear Analysis: Real World Applications, vol. 13, no. 5, pp. 2259-2270, 2012.

[34] C.-D. Zheng, Y. Wang, and Z. Wang, "Stability analysis of stochastic fuzzy Markovian jumping neural networks with leakage delay under impulsive perturbations," Journal of the Franklin Institute, vol. 351, no. 3, pp. 1728-1755, 2014.

[35] M. Dong, H. Zhang, and Y. Wang, "Dynamics analysis of impulsive stochastic Cohen-Grossberg neural networks with Markovian jumping and mixed time delays," Neurocomputing, vol. 72, no. 7-9, pp. 1999-2004, 2009.

[36] R. Rakkiyappan and P. Balasubramaniam, "Dynamic analysis of Markovian jumping impulsive stochastic Cohen-Grossberg neural networks with discrete interval and distributed timevarying delays," Nonlinear Analysis: Hybrid Systems, vol. 3, no. 4, pp. 408-417, 2009.

[37] H. Jiang and J. Liu, "Dynamics analysis of impulsive stochastic high-order BAM neural networks with Markovian jumping and mixed delays," International Journal of Biomathematics, vol. 4, no. 2, pp. 149-170, 2011.

[38] Q. Zhu and J. Cao, "Stability analysis of Markovian jump stochastic BAM Neural networks with impulse control and mixed time delays," IEEE Transactions on Neural Networks and Learning Systems, vol. 23, no. 3, pp. 467-479, 2012.

[39] S.-L. Wu, K.-L. Li, and J.-S. Zhang, "Exponential stability of discrete-time neural networks with delay and impulses," Applied Mathematics and Computation, vol. 218, no. 12, pp. 6972-6986, 2012.
[40] S. L. Wu, K.-L. Li, and T. Z. Huang, "Exponential stability of static neural networks with time delay and impulses," IET Control Theory \& Applications, vol. 5, no. 8, pp. 943-951, 2011.

[41] S.-L. Wu, K.-L. Li, and T.-Z. Huang, "Global exponential stability of static neural networks with delay and impulses: discrete-time case," Communications in Nonlinear Science and Numerical Simulation, vol. 17, no. 10, pp. 3947-3960, 2012.

[42] J. Lu, D. W. C. Ho, and J. Cao, "A unified synchronization criterion for impulsive dynamical networks," Automatica, vol. 46, no. 7, pp. 1215-1221, 2010.

[43] W.-H. Chen and W. X. Zheng, "Global exponential stability of impulsive neural networks with variable delay: an LMI approach," IEEE Transactions on Circuits and Systems I: Regular Papers, vol. 56, no. 6, pp. 1248-1259, 2009.

[44] W. Zhang, Y. Tang, X. Wu, and J.-A. Fang, "Synchronization of nonlinear dynamical networks with heterogeneous impulses," IEEE Transactions on Circuits and Systems I: Regular Papers, vol. 61, no. 4, pp. 1220-1228, 2014.

[45] W. Zhang, Y. Tang, J.-A. Fang, and X. Wu, "Stability of delayed neural networks with time-varying impulses," Neural Networks, vol. 36, pp. 59-63, 2012.

[46] W. K. Wong, W. Zhang, Y. Tang, and X. Wu, "Stochastic synchronization of complex networks with mixed impulses," IEEE Transactions on Circuits and Systems I: Regular Papers, vol. 60, no. 10, pp. 2657-2667, 2013.

[47] D. J. Higham, "An algorithmic introduction to numerical simulation of stochastic differential equations," SIAM Review, vol. 43 , no. 3, pp. 525-546, 2001.

[48] H. K. Khalil, Nonlinear Systems, Prentice Hall, Upper Saddle River, NJ, USA, 1996.

[49] Q. Zhu and J. Cao, "Robust exponential stability of markovian jump impulsive stochastic Cohen-Grossberg neural networks with mixed time delays," IEEE Transactions on Neural Networks, vol. 21, no. 8, pp. 1314-1325, 2010.

[50] K. Gu, "An integral inequality in the stability problem of time-delay systems," in Proceedings of the 39th IEEE Confernce on Decision and Control, pp. 2805-2810, Sydney, Australia, December 2000.

[51] Y. Wang, L. Xie, and C. E. de Souza, "Robust control of a class of uncertain nonlinear systems," Systems and Control Letters, vol. 19, no. 2, pp. 139-149, 1992.

[52] T. H. Gronwall, "Note on the derivatives with respect to a parameter of the solutions of a system of differential equations," Annals of Mathematics, vol. 20, no. 4, pp. 292-296, 1919.

[53] A. Halanay and J. A. Yorke, "Some new results and problems in the theory of differential-delay equations," SIAM Review, vol. 13, pp. 55-80, 1971.

[54] A. Seuret and F. Gouaisbaut, "Wirtinger-based integral inequality: application to time-delay systems," Automatica, vol. 49, no. 9, pp. 2860-2866, 2013.

[55] P. G. Park, J. W. Ko, and C. Jeong, "Reciprocally convex approach to stability of systems with time-varying delays," Automatica, vol. 47, no. 1, pp. 235-238, 2011.

[56] Z. Wang, Y. Liu, and X. Liu, "Exponential stabilization of a class of stochastic system with markovian jump parameters and mode-dependent mixed time-delays," IEEE Transactions on Automatic Control, vol. 55, no. 7, pp. 1656-1662, 2010.

[57] H. Huang, T. Huang, and X. Chen, "A mode-dependent approach to state estimation of recurrent neural networks with Markovian jumping parameters and mixed delays," Neural Networks, vol. 46, pp. 50-61, 2013. 
[58] X. Yang, J. Cao, and J. Lu, "Synchronization of randomly coupled neural networks with markovian jumping and timedelay," IEEE Transactions on Circuits and Systems I: Regular Papers, vol. 60, no. 2, pp. 363-376, 2013.

[59] A. V. Skorohod, Asymptotic Methods in the Theory of Stochastic Differential Equations, American Mathematical Society, 2009.

[60] S. Boyd, L. El Ghaoui, E. Feron et al., Linear Matrix Inequalities in System and Control Theory, SIAM, Philadelphia, Pa, USA, 1994. 


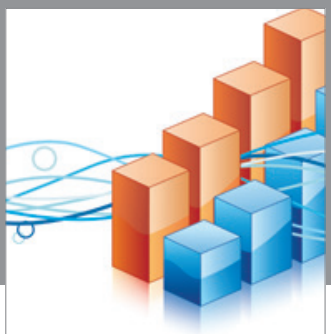

Advances in

Operations Research

vatem alat4

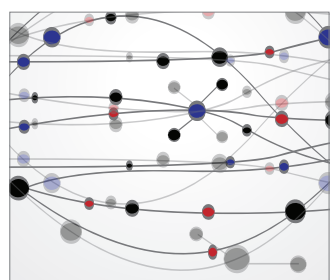

\section{The Scientific} World Journal
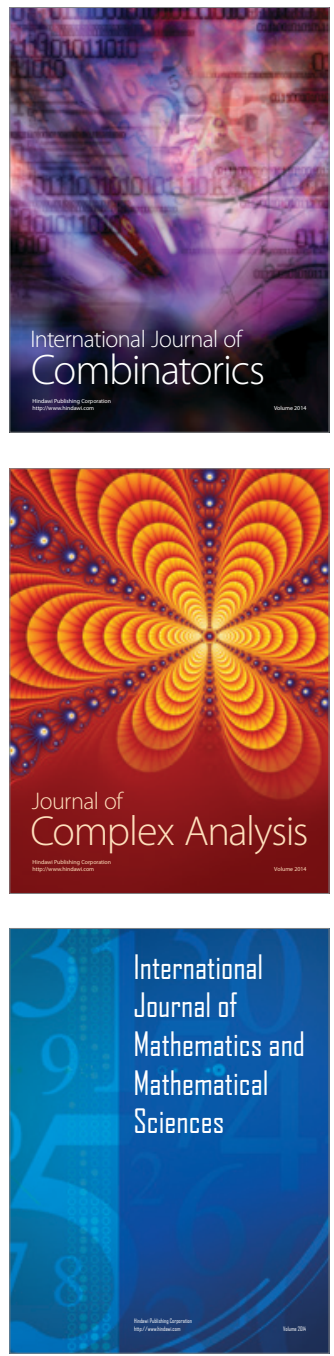
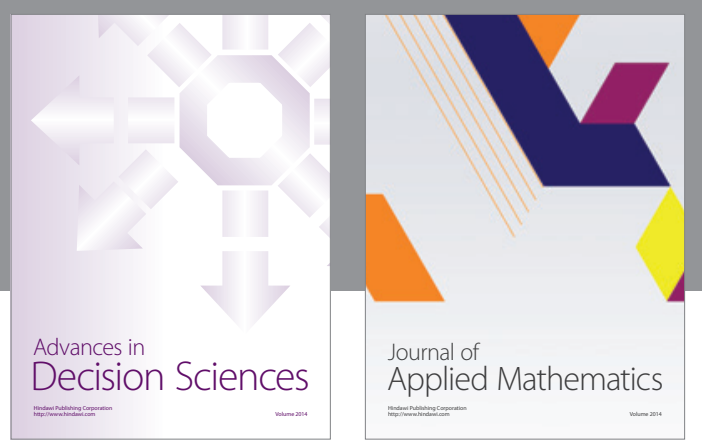

Algebra

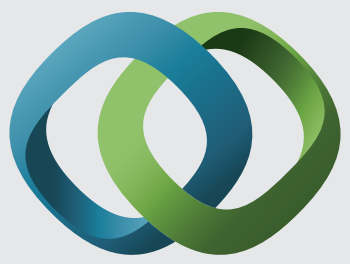

\section{Hindawi}

Submit your manuscripts at

http://www.hindawi.com
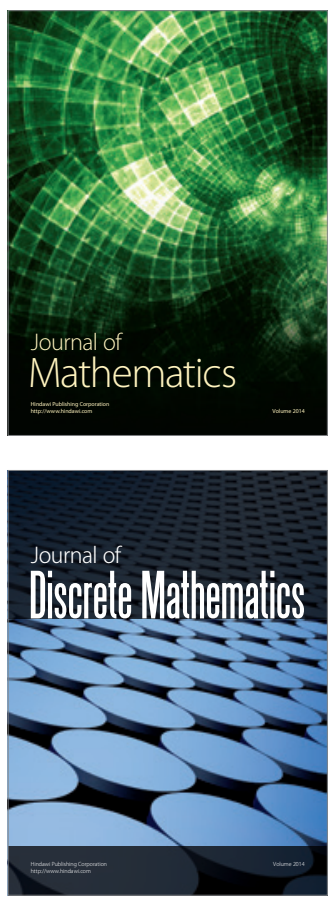

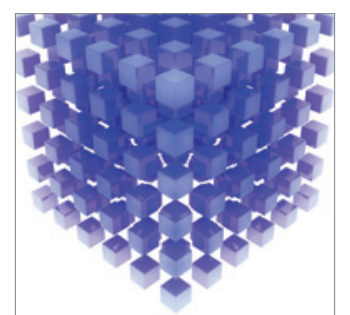

Mathematical Problems in Engineering
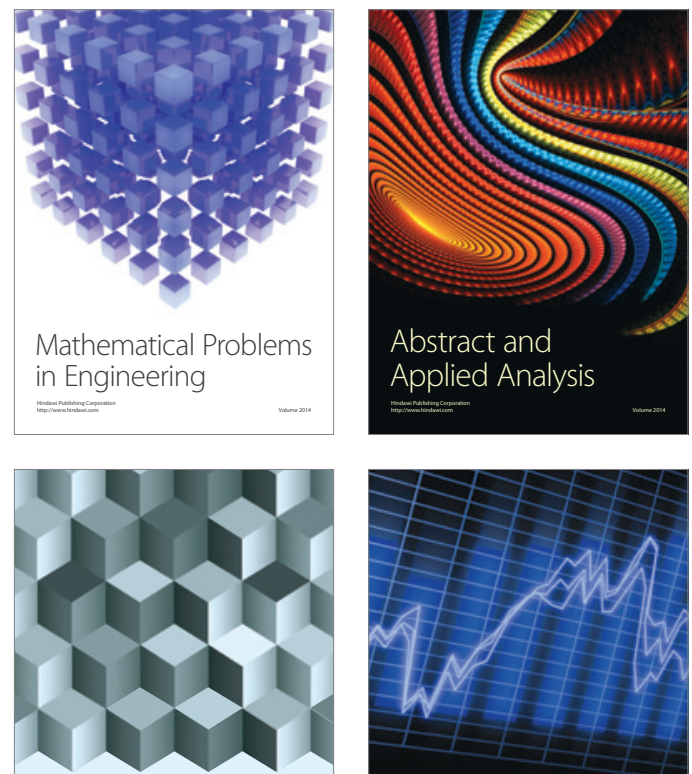

Journal of

Function Spaces

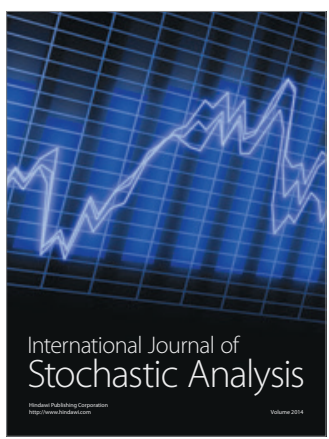

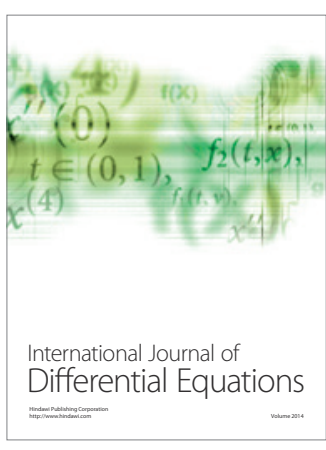
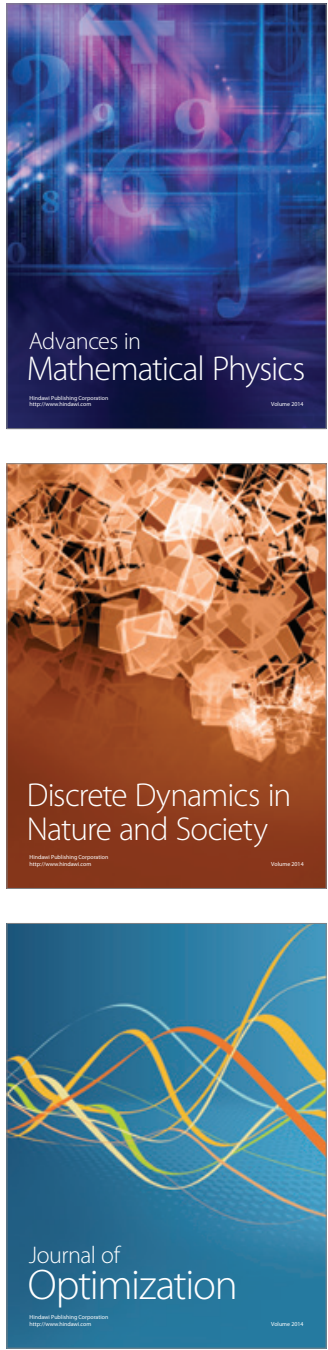